\title{
QUEEN'S
UNIVERSITY
BELFAST
}

\section{Kinetostatic-model-based Stiffness Analysis of Exechon PKM}

Zhang, J., Zhao, Y., \& Jin, Y. (2016). Kinetostatic-model-based Stiffness Analysis of Exechon PKM. Robotics and Computer-Integrated Manufacturing, 37, 208-220. https://doi.org/10.1016/j.rcim.2015.04.008

Published in:

Robotics and Computer-Integrated Manufacturing

Document Version:

Peer reviewed version

Queen's University Belfast - Research Portal:

Link to publication record in Queen's University Belfast Research Portal

\section{Publisher rights}

Copyright 2015 Elsevier.

This manuscript is distributed under a Creative Commons Attribution-NonCommercial-NoDerivs License

(https://creativecommons.org/licenses/by-nc-nd/4.0/), which permits distribution and reproduction for non-commercial purposes, provided the author and source are cited.

\section{General rights}

Copyright for the publications made accessible via the Queen's University Belfast Research Portal is retained by the author(s) and / or other copyright owners and it is a condition of accessing these publications that users recognise and abide by the legal requirements associated with these rights.

Take down policy

The Research Portal is Queen's institutional repository that provides access to Queen's research output. Every effort has been made to ensure that content in the Research Portal does not infringe any person's rights, or applicable UK laws. If you discover content in the Research Portal that you believe breaches copyright or violates any law, please contact openaccess@qub.ac.uk. 


\section{Kinetostatic-model-based Stiffness Analysis of}

\author{
Jun Zhang \\ School of Mechanical Engineering, \\ Anhui University of Technology, \\ Ma'anshan 243032, China \\ * Corresponding author. \\ email: zhang_jun_81@163.com
}

Yanqin Zhao

School of Mechanical Engineering, Anhui University of Technology, Ma'anshan 243032, China email: zhaoyanqin_91@163.com

Yan Jin

School of Mechanical and Aerospace

Engineering,

Queen's University Belfast,

BT9 5HN, Northern Ireland, UK email: y.jin@qub.ac.uk

Keywords:

PKM

Exechon

Kinetostatics

Stiffness modeling

Substructure synthesis

\section{Exechon PKM}

As a comparative newly-invented PKM with over-constraints in kinematic chains, the Exechon has attracted extensive attention from the research society. Different from the well-recognized kinematics analysis, the research on the stiffness characteristics of the Exechon still remains as a challenge due to the structural complexity. In order to achieve a thorough understanding of the stiffness characteristics of the Exechon PKM, this paper proposed an analytical kinetostatic model by using the substructure synthesis technique. The whole PKM system is decomposed into a moving platform subsystem, three limb subsystems and a fixed base subsystem, which are connected to each other sequentially through corresponding joints. Each limb body is modeled as a spatial beam with uniform cross-section constrained by two sets of lumped springs. The equilibrium equation of each individual limb assemblage is derived through finite element formulation and combined with that of the moving platform derived with Newtonian method to construct the governing kinetostatic equations of the system after introducing the deformation compatibility conditions between the moving platform and the limbs. By extracting the $6 \times 6$ block matrix from the inversion of the governing compliance matrix, the stiffness of the moving platform is formulated. The computation for the stiffness of the Exechon PKM at a typical configuration as well as throughout the workspace is carried out in a quick manner with a piece-by-piece partition algorithm. The numerical simulations reveal a strong position-dependency of the PKM's stiffness in that it is symmetric relative to a work plane due to structural features. At the last stage, the effects of some design variables such as structural, dimensional and stiffness parameters on system rigidity are investigated with the purpose of providing useful information for the structural optimization and performance enhancement of the Exechon PKM. It is worthy mentioning that the proposed methodology of stiffness modeling in this paper can also be applied to other overconstrained PKMs and can evaluate the global rigidity over workplace efficiently with minor revisions.

\section{Introduction}

Compared with their counterparts of traditional serial kinematic machine (SKM) tools, parallel kinematic machines (PKMs) with lower mobility claim the advantages of high stiffness, small moving mass and compact volume by utilizing the parallel arrangements of motion system. This makes PKMs with lower mobility a promising alternative solution for high-speed machining (HSM) of extra large scale components with complicated geometries. For instance, the commercial success of Sprint Z3 head has applied in aeronautical industries $[1,2]$. Another commercial attemption was the use of Tricept robots in automotive industries, which however has seen little acceptance so far due to technical reasons [3-6]. Other propositions of using PKMs for HSM can also be traced in recent publications [7-10]. More recently, the Exechon PKM has been proposed and patented by Neumann under the motivation of reducing the number of passive joints and non-actuated degree of freedom [11]. The prototyping system has been developed and its improved performance has been demonstrated through primary experiments [12].

As one of the most overwhelming concerns in the early design stage of such a PKM designed for HSM applications where high rigidity and high positioning accuracy are required, stiffness has attracted extensive attention from the research societies [13-22]. Among all the studies towards the stiffness modeling and evaluation, the finite element method (FEM) [13,14], the matrix structure method (MSM) [15,16], the virtual joint method (VJM) [17,18] and the screw-based method (SBM) [19-22] are the most common used approaches. For example, Pairs proposed a FE model for a planar parallel manipulator with flexible links and analysed the system dynamics [13]. Huang et al. proposed a stiffness model for a tripod-based PKM by decomposing the overall system into two separate substructures and 
formulating the stiffness expressions of each substructure with virtual work principle [16]. A similar model of the 3-DOF CaPaMan parallel manipulator is established by Ceccarelli and Carbone who considered the kinematic and static features of the three legs in view of the motions of every joint and link [17]. Li and Xu proposed an intuitive method based upon an overall Jacobian to formulate the stiffness matrix of a 3-PUU translational PKM. In their model, the compliances subjected to both actuations and constraints are considered and the overall stiffness matrix of the lower mobility parallel manipulator can be derived intuitively [19]. Following the same track, Huang and Liu et al proposed a stiffness modelling approach for the lower mobility parallel manipulators using the generalized Jacobian [20].

Different from abundant investigations on the stiffness of the PKMs as mentioned above, the studies focusing on the stiffness of the Exechon PKM are quite scare. Bonnemains and co-workers [23] derived a static model for Tripteor by taking into account the nonlinear compliances of joints and legs, with which the static behaviour of the system was analyzed. They then extended the static model to a dynamic one with the energy method [24]. This dynamic model was then used to study the impact of component deformations on the generated surfaces. Li et al [25] established an analytical stiffness model for Exechon X150 with the screw theory and the virtual work principle. Based on the proposed model, the mechanism's stiffness at typical configurations was analysed and compared with FE simulations. More recently, Bi [26] formulated a stiffness matrix for the Exechon X700 PKM by using the kinetostatic method. Based on kinematic Jacobian matrices, the impacts of stiffness from both axial and torsional compliances of actuated legs were considered and the distributions of stiffness in each individual direction were predicted.

It is worthy to mention that a lack of consideration of the compliance of limb body as well as the orientation-dependency of the spherical joint of the Exechon PKM can be traced in most of the above studies. Although the effects of these factors can be included if a FE model is applied, however, adopting the FEM to analyze the stiffness of the Exechon PKM seems to be a second priority choice in that it might require reconstruction or re-meshing of kinematic chain assemblages when the PKM's configuration is changed within the working envelope.

The present work has a different perspective in that it aims to achieve a thorough understanding of the stiffness characterisitics of the Exechon PKM, which can be summarized in two aspects: (1) To predict the Exechon stiffness not only at typical configurations but also throughout the workspace accurately in a computational-effective manner. (2) To figure out the influences of major design variables and quantify the contribution of each individual compliant component to the global stiffness. For this purpose, a kinetostatic model for the Exechon PKM is developed by considering the flexibilities of both actuated and passive joints as well as limb structures. With the proposed kinetostatic model, the stiffness of the PKM is computed followed by a parameter study to provide useful information for the structural optimization and rigidity enhancement. The reminder of the paper is organized as follows. Firstly, the structure of the Exechon module and its inverse kinematics are briefly described in Section 2. Secondly, a kinetostatic stiffness model is established using the technique of substructure synthesis in Section 3. Then, a parametric analysis is conducted in Section 4. Finally, the research work in this paper is summarized and conclusions are drawn in Section 5.

\section{Kinematic Modeling}

A CAD model for the Exechon module is shown in Fig. 1.

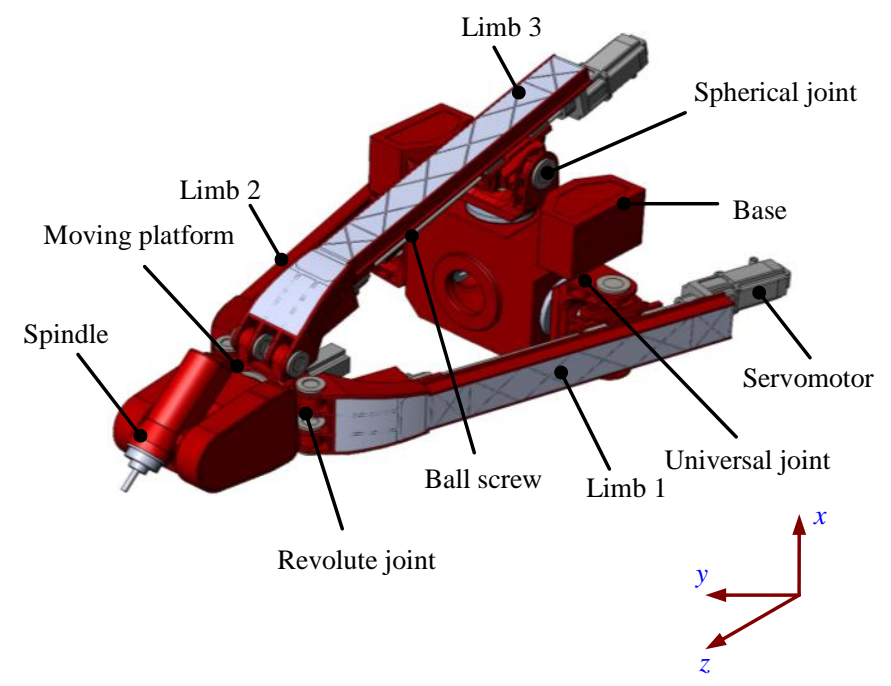

Fig. 1. Structure of the Exechon module 
As shown in Fig. 1, the Exechon module consists of a moving platform, a fixed base and three kinematic limbs. Limb 1 and limb 2 are symmetrical with respect to limb 3. Limb 1 (and 2) connects the base to the platform by a universal (U) joint followed by a prismatic (P) joint and a revolute $(\mathrm{R})$ joint in sequence, where the $\mathrm{P}$ joint is driven by a lead screw linear actuator. The constitution of limb 3 is slightly different from limb 1 and limb 2 in that it connects to the moving platform by a spherical (S) joint. An electrical spindle can be mounted on the platform to implement high-speed milling. Driven independently by three servomotors, one translation along $z$ axis and two rotations about $x$ and $y$ axes can be achieved.

For the convenience of analysis, a schematic diagram of the Exechon module is depicted in Fig. 2. Herein, $C_{i}$ ( $i=1 \sim 3$ ) denotes the rear end of the $i^{\text {th }} \operatorname{limb} ; A_{1}$ and $A_{2}$ are the centers of universal joints in limb 1 and limb $2 ; A_{3}$ is the center of the spherical joint in $\operatorname{limb} 3 ; B_{i}(i=1 \sim 3)$ denotes the geometric center of the $i^{\text {th }}$ revolute joint, respectively. $\triangle A_{1} A_{2} A_{3}$ and $\triangle B_{1} B_{2} B_{3}$ are assumed to be isosceles right triangles, and $\angle A_{3}=\angle B_{3}=90^{\circ}$.

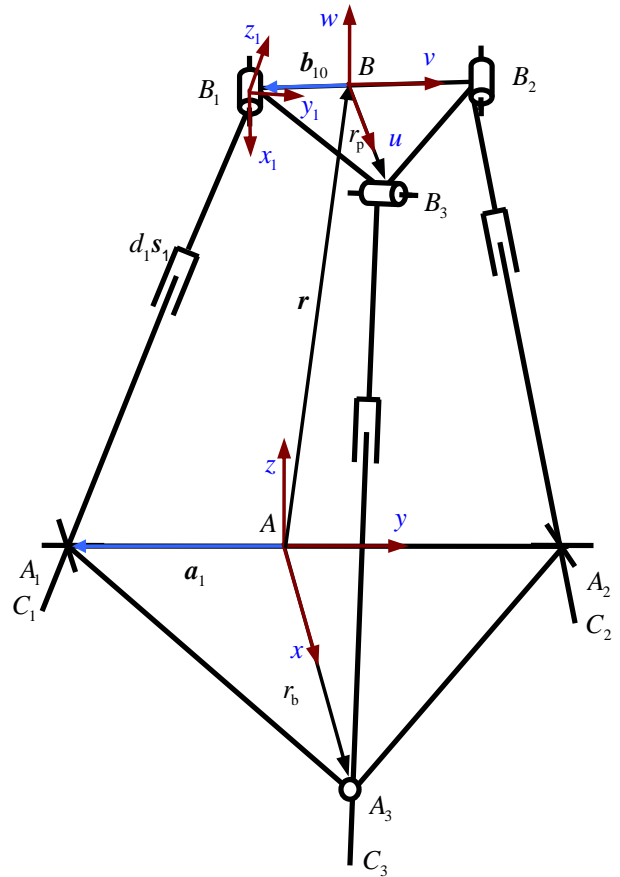

Fig. 2. Schematic diagram of the Exechon module

To facilitate the formulation, Cartesian coordinate systems are set as follows. The global coordinate system $A-x y z$ is attached to the base, where $A$ is the center point of $A_{1} A_{2}$. The $x$ axis is parallel to $A A_{3}$ and the $y$ axis is parallel to $A_{1} A_{2}$ while the $z$ axis is determined by the right-hand rule. The body-fixed coordinate system $B-u v w$ is placed at the center point $B$ of $B_{1} B_{2}$, with the $u$ axis parallel to $B B_{3}$, the $v$ axis parallel to $B B_{2}$ and $w$

perpendicular to $\triangle B_{1} B_{2} B_{3}$. The limb reference frame $B_{i}-x_{i} y_{i} z_{i}$ is established at the center point $B_{i}$, with $z_{i}$ coincident with the limb. Herein, $x_{i}(i=1 \sim 3)$ is parallel to the axis of the $i^{\text {th }}$ revolute joint, while $y_{i}(i=1 \sim 3)$ is decided by the right-hand rule. For clarity, only one limb reference frame in limb 1 is depicted in Fig. 2.

The transformation matrix $\boldsymbol{R}_{0}$ of the frame $B-u v w$ with respect to the frame $A-x y z$ can be formulated as

$\boldsymbol{R}_{0}=\left[\begin{array}{ccc}\mathrm{c} \varphi \mathrm{c} \theta & \mathrm{c} \varphi \mathrm{s} \theta s \psi-\mathrm{s} \varphi \mathrm{c} \psi & \mathrm{c} \varphi \mathrm{s} \theta \mathrm{c} \psi+\mathrm{s} \varphi \mathrm{s} \psi \\ \mathrm{s} \varphi \mathrm{c} \theta & \mathrm{s} \varphi \mathrm{s} \theta \mathrm{s} \psi+\mathrm{c} \varphi \mathrm{c} \psi & \mathrm{s} \varphi \mathrm{s} \theta \mathrm{c} \psi-\mathrm{c} \varphi \mathrm{s} \psi \\ -\mathrm{s} \theta & \mathrm{c} \theta \mathrm{s} \psi & \mathrm{c} \theta \mathrm{c} \psi\end{array}\right]$

where "s" and "c" denote "sin" and "cos" functions, respectively; $\psi, \theta$ and $\varphi$ are Euler angles in terms of precession, nutation and rotation.

As shown in Fig. 2, the position vector of point $B_{i}$ measured in the $A-x y z$ can be given as

$\boldsymbol{b}_{i}=\boldsymbol{R}_{0} \boldsymbol{b}_{i 0}+\boldsymbol{r}=\boldsymbol{a}_{i}+d_{i} \boldsymbol{s}_{i}$

where $\boldsymbol{r}$ and $\boldsymbol{a}_{i}$ represent the vectors of points $B$ and $A_{i}$ measured in the $A-x y z$ frame, respectively; $\boldsymbol{b}_{i 0}$ represents the vector of point $B_{i}$ measured in the $B-u v w$ frame; $d_{i}$ denotes the distance between $A_{i}$ and $B_{i} ; \boldsymbol{s}_{i}$ is unit vector of the $i^{\text {th }} \operatorname{limb}$. And there exist

$$
\boldsymbol{a}_{i}=r_{\mathrm{b}}\left[\begin{array}{c}
\cos \beta_{i} \\
\sin \beta_{i} \\
0
\end{array}\right], \quad \boldsymbol{b}_{i 0}=r_{\mathrm{p}}\left[\begin{array}{c}
\cos \beta_{i} \\
\sin \beta_{i} \\
0
\end{array}\right], \boldsymbol{r}=\left[\begin{array}{c}
p_{x} \\
p_{y} \\
p_{z}
\end{array}\right]
$$

where $r_{\mathrm{p}}, r_{\mathrm{b}}$ are the radii of the platform and the base, respectively; $\beta_{i}(i=1 \sim 3)$ is the position angle of a joint, and $\beta_{1}=-\pi / 2, \beta_{2}=-3 \pi / 2, \beta_{3}=0 ; p_{x}, p_{y}$ and $p_{z}$ are coordinates of point $B$ measured in $A-x y z$.

Taking $\psi, \theta$ and $p_{z}$ as independent coordinates, and considering the constrains of revolute joints, one can obtain the followings

$$
\left\{\begin{array}{l}
\varphi=0 \\
p_{x}=p_{z} \tan \theta \\
p_{y}=\left(\left(p_{x}-r_{b}\right) \sin \theta+p_{z} \cos \theta\right) \tan (-\psi)
\end{array}\right.
$$

Eq. (4) is the parasitic motions of the Exechon module. The inverse position analysis can be conducted as follows:

$$
\begin{aligned}
& d_{i}=\left\|\boldsymbol{b}_{i}-\boldsymbol{a}_{i}\right\| \\
& \boldsymbol{s}_{i}=\frac{\boldsymbol{b}_{i}-\boldsymbol{a}_{i}}{d_{i}}=\left[\begin{array}{lll}
s_{i x} & s_{i y} & s_{i z}
\end{array}\right]^{\mathrm{T}}
\end{aligned}
$$

\section{Kinetostatic Modeling}


In this section, the substructure synthesis technique is applied to formulate the kinetostatic equations of the Exechon module based on which the stiffness expressions of the Exechon PKM can be extracted. Due to the structural complexity of the Exechon, the following hypotheses and approximations are made to facilitate the analytical derivation:

(1) The base and the platform are treated as rigid bodies for their high rigidities;

(2) The limb body is modeled as a hollowed spatial beam with a constant rectangular cross-section according to its structural feature;

(3) The revolute, universal and spherical joints are simplified to virtual lumped springs with equivalent stiffness coefficients at their geometric centers which can be calculated through FE computation or semi-analytical method [27].

(4) The frictions and inertial forces as well as damping effects are neglected though they can be easily integrated into the governing equations of the system.

3.1. Stiffness formulation and equilibrium equation derivation of an individual limb assemblage

The assemblage of an individual UPR/SPR limb in the Exechon module is shown in Fig. 3.
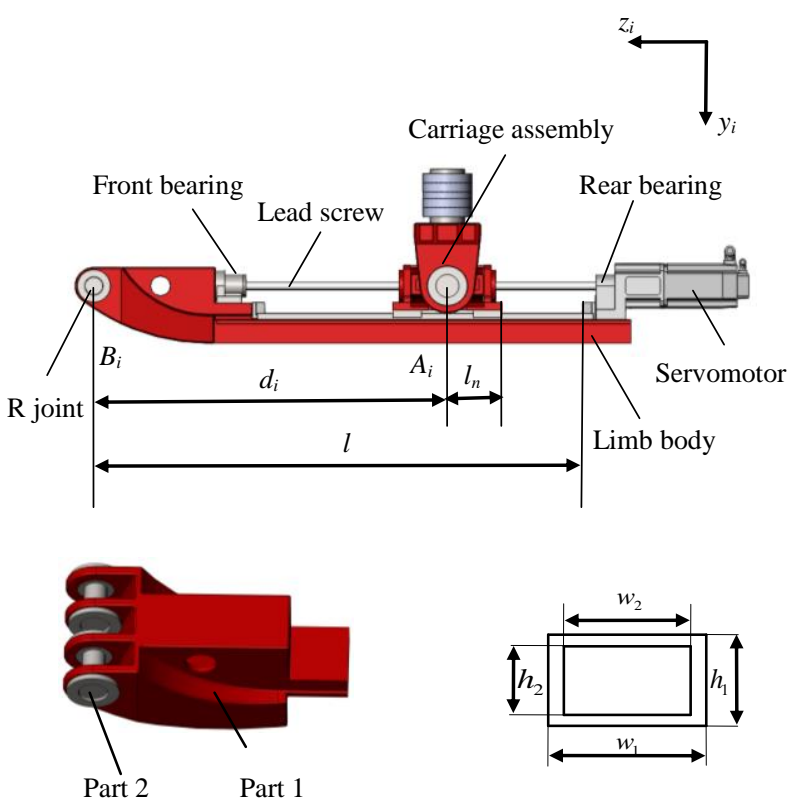

Constitution of $\mathrm{R}$ joint

Cross-section

Fig. 3. Assembly of a UPR/SPR limb
According to the assembling relationships and structural features of the limb, one can classify all the components in an individual limb into three categories when formulating the stiffness expression: (1) the limb body; (2) the R joint (including part 1 and part 2); (3) the U/S joint (including lead-screw assembly, guideway assembly and carriage assembly). Since all the joints in the limb assemblage are simplified into virtual lumped springs with equivalent stiffness, the UPR/SPR limb can thus be modelled as a uniform spatial beam constrained by two sets of lumped springs as shown in Fig. 4.

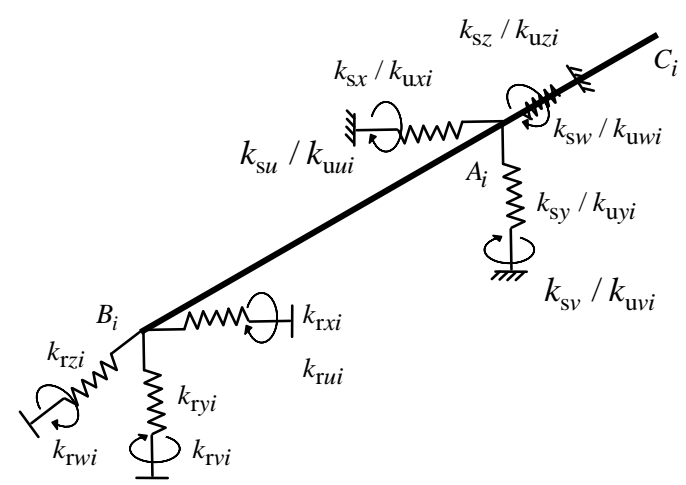

Fig. 4. Simplified force diagram of an individual limb

Herein, $k_{\mathrm{s} x}, k_{\mathrm{s} y}, k_{\mathrm{s} z}$ and $k_{\mathrm{s} u}, k_{\mathrm{s} v}, k_{\mathrm{s} w}$ are the equivalent spring constants of three translational and three torsional virtual springs of the spherical joint in limb 3. Similarly, $k_{\mathrm{u} x i}, k_{\mathrm{u} y i}, k_{\mathrm{u} z i}$ and $k_{\mathrm{u} u i}, k_{\mathrm{u} v i}, k_{\mathrm{u} w i}$ are the equivalent spring constants of three translational and three torsional virtual springs of the universal joint in limb $i(i=1,2) ; k_{\mathrm{r} x i}, k_{\mathrm{ry} i}$, $k_{\mathrm{r} z i}$ and $k_{\mathrm{r} u i}, k_{\mathrm{r} v i}, k_{\mathrm{r} w i}$ are the equivalent spring constants of three translational and three torsional virtual springs of the revolute joint in the $i^{\text {th }}$ limb assembly, respectively. With the consideration of structural constraint features, the values of the spring constants of $k_{\mathrm{s} u}, k_{\mathrm{s} v}, k_{\mathrm{s} w}, k_{\mathrm{u} u i}, k_{\mathrm{uv} i}$, $k_{\text {rui }}$ are set to be zero.

As shown in Fig. 3, a revolute joint consists of two components denoted as Part 1(including rotors and bearings) and Part 2(one complex entity). Assume the stiffness of Part 1 is defined as

$\boldsymbol{k}_{\mathrm{rP} 1}=\operatorname{diag}\left[\begin{array}{llllll}k_{\mathrm{r} 1 x} & k_{\mathrm{r} 1 y} & k_{\mathrm{r} 1 z} & k_{\mathrm{r} 1 u} & k_{\mathrm{r} 1 v} & k_{\mathrm{r} 1 w}\end{array}\right]$

where $k_{\mathrm{r} 1 x}, k_{\mathrm{r} 1 y}, k_{\mathrm{r} 1 z}$ and $k_{\mathrm{r} 1 u}, k_{\mathrm{r} 1 v} k_{\mathrm{r} 1 w}$ are three linear stiffness coefficients and three angular stiffness coefficients along and about three perpendicular axes at the mass center of Part 1 , which can be determined through the commercial software ANSYS. Note that $k_{\mathrm{r} 1 u}=0$ according to its kinematic constraint features. In a similar way, the stiffness of Part 2 can be denoted as 


$$
\boldsymbol{k}_{\mathrm{rP} 2}=\operatorname{diag}\left[\begin{array}{lllllll}
k_{\mathrm{r} 2 x} & k_{\mathrm{r} 2 y} & k_{\mathrm{r} 2 z} & k_{\mathrm{r} 2 u} & k_{\mathrm{r} 2 v} & k_{\mathrm{r} 2 w}
\end{array}\right]
$$

where $k_{\mathrm{r} 2 x}, k_{\mathrm{r} 2 y}, k_{\mathrm{r} 2 z}$ and $k_{\mathrm{r} 2 u}, k_{\mathrm{r} 2 v} k_{\mathrm{r} 2 w}$ are three linear stiffness coefficients and three angular stiffness coefficients along and about three perpendicular axes at the centroid of Part 2.

Therefore, the stiffness of a revolute joint can be regarded as a serial aggregation of Part 1 and Part 2

$$
\boldsymbol{k}_{\mathrm{ri} i}=\left(\boldsymbol{k}_{\mathrm{rP} 1}^{-1}+\boldsymbol{k}_{\mathrm{rP} 2}^{-1}\right)^{-1}=\operatorname{diag}\left[\begin{array}{lllllll}
k_{\mathrm{r} x i} & k_{\mathrm{r} y i} & k_{\mathrm{r} z i} & k_{\mathrm{r} v i} & k_{\mathrm{r} w i}
\end{array}\right]
$$

A universal joint is composed of lead-screw assembly (including front bearing, lead screw and rear bearing), guideway assembly and carriage assembly. Therefore, the stiffness of the universal joint $\boldsymbol{k}_{\mathrm{u} i}$ can be calculated through the superposition of each part as addressed. The expression of $\boldsymbol{k}_{\mathrm{u} i}$ can be formulated as

$$
\boldsymbol{k}_{\mathrm{u} i}=\left(\boldsymbol{k}_{\mathrm{ul}}^{-1}+\boldsymbol{k}_{\mathrm{ug}}^{-1}+\boldsymbol{k}_{\mathrm{uc}}^{-1}\right)^{-1}
$$

where $\boldsymbol{k}_{\mathrm{ul}}, \boldsymbol{k}_{\mathrm{ug}}$ and $\boldsymbol{k}_{\mathrm{uc}}$ are the stiffness of lead-screw assembly, guideway assembly and the carriage assembly, respectively.

The lead-screw assembly is serially comprised of the front bearing, the lead screw and the rear bearing, which only provides a constraint to the carriage in axial direction. Thus, its stiffness can be expressed as

$$
\boldsymbol{k}_{\mathrm{ul}}=\operatorname{diag}\left[\begin{array}{llllll}
0 & 0 & k_{\mathrm{ul} z} & 0 & 0 & 0
\end{array}\right]
$$

where $k_{\mathrm{ulz}}$ is the stiffness of the lead-screw assembly in the axial direction, and

$$
k_{\mathrm{ulz}}=\left[\left(l-d_{i}-l_{n}\right) / E A+k_{\mathrm{F}}^{-1}+k_{\mathrm{R}}^{-1}\right]^{-1}
$$

where $l$ is the length of limb body; $l_{n}$ is half of the width of the carriage assembly; $E$ is the Yong's modulus of the lead screw; $A$ is its cross-sectional area; $k_{\mathrm{F}}$ and $k_{\mathrm{R}}$ are the axial stiffness of the front bearing and the rear bearing, respectively. They can be determined from bearing datasheets and finite element analysis.

The guideway assembly can be simplified into a virtual lumped spring with stiffness in six directions. Apparently, this virtual lumped spring does not provide constraints to the carriage along the axial direction of the guideway. Therefore, the stiffness of the guideway assembly can be formulated as

$$
\boldsymbol{k}_{\mathrm{ug}}=\operatorname{diag}\left[k_{\mathrm{ug} x} k_{\mathrm{ug} y} k_{\mathrm{ug} z} k_{\mathrm{ug} u} k_{\mathrm{ug} v} k_{\mathrm{ug} w}\right]
$$

where $k_{\text {ugz }}=0 . k_{\text {ugg }}, k_{\text {ugy }}$ and $k_{\text {ugz }}$ are three translational stiffness coefficients along three Cartesian axes of $x, y$ and $z ; k_{\text {ugu }}, k_{\text {ugv }}$ and $k_{\text {ugw }}$ are three rotational stiffness about three Cartesian axes of $x, y$ and $z$. The coefficients in Eq. (13) can be determined through finite element analysis and semi-analytical fitting.

Similarly, the stiffness matrix of the carriage assembly $\boldsymbol{k}_{\mathrm{uc}}$ can be expressed as

$$
\boldsymbol{k}_{\mathrm{uc}}=\operatorname{diag}\left[k_{\mathrm{ucx}} k_{\mathrm{ucy}} k_{\mathrm{ucz}} k_{\mathrm{ucu}} k_{\mathrm{ucv}} k_{\mathrm{uc} w}\right]
$$

where $k_{\text {ucz }}=k_{\text {ucu }}=k_{\mathrm{uc} v}=0 . \quad k_{\mathrm{uc} x}, \quad k_{\mathrm{ucy}}$ and $k_{\mathrm{ucz}}$ are three translational stiffness coefficients along three Cartesian axes of $x, y$ and $z ; k_{\mathrm{uc} u}, k_{\mathrm{ucv}}$ and $k_{\mathrm{uc} w}$ are three rotational stiffness about three Cartesian axes of $x, y$ and $z$. Similar to the guideway assembly, coefficients in Eq. (14) can be determined through finite element analysis and semi-analytical fitting.

Substituting Eqs. (11) (14) into Eq. (10), one can formulate the stiffness of universal joint assembly as

$\boldsymbol{k}_{\mathrm{u} i}=\operatorname{diag}\left[k_{\mathrm{u} x i} k_{\mathrm{u} y i} k_{\mathrm{u} z i} \quad 00 k_{\mathrm{uw} i}\right]$

The derivation of stiffness of a spherical joint is the same to a revolute joint except stiffness of the carriage in that the values of the stiffness coefficients about three perpendicular axes are set to be zero.

Therefore, the stiffness matrix of the spherical joint $\boldsymbol{k}_{\mathrm{s}}$ in limb 3 can be denoted as

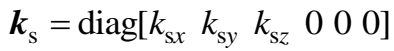

The simplified spatial beam shown in Fig. 4 can be meshed into finite elements with each node having three linear and three angular coordinates along and about three axes. Fig. 5 shows the $e^{\text {th }}$ element of the $i^{\text {th }}$ limb in element reference frame $N_{i}^{e}-x_{i}^{e} y_{i}^{e} z_{i}^{e}$.

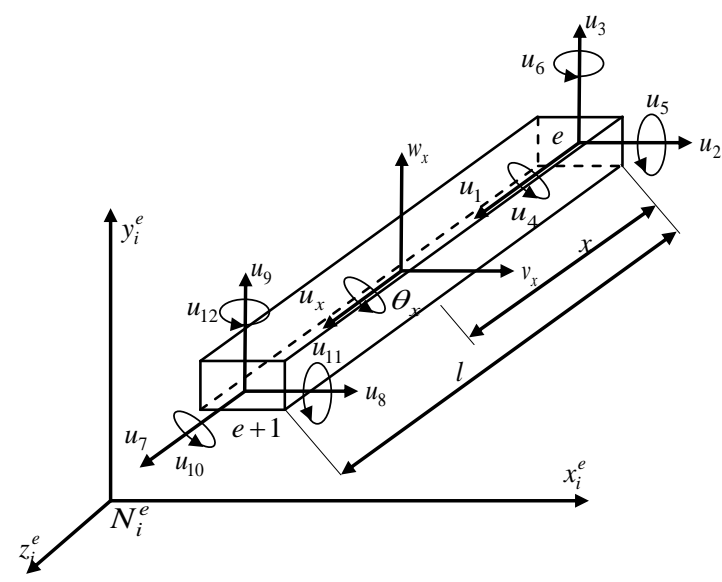

Fig. 5. Definition of spatial beam element 
Herein, $e$ and $e+1$ denote two adjacent nodes of the element; $u_{i}(i=1 \sim 12)$ represents the nodal coordinates and the frame of $N_{i}^{e}-x_{i}^{e} y_{i}^{e} z_{i}^{e}$ is parallel to the limb frame $B_{i}-x_{i} y_{i} z_{i}$

To simplify the formulation, each limb body is discretized into $n$ elements with $B_{i}, A_{i}$ and $C_{i}$ being nodes of elements. As a result, a set of equilibrium equations of the $i^{\text {th }}$ limb in frame $B_{i}-x_{i} y_{i} z_{i}$ can be formulated with adequate boundary conditions and can be expressed as

$$
\boldsymbol{k}_{i} \boldsymbol{u}_{i}=\boldsymbol{f}_{i}
$$

where $\boldsymbol{k}_{i}$ is the stiffness matrix of each limb body; $\boldsymbol{u}_{i}$ and $f_{i}$ are the general coordinates vector and external loads vector of the $i^{\text {th }}$ limb body and can be expressed as

$$
\left\{\begin{array}{l}
\boldsymbol{u}_{i}=\left[\begin{array}{llllllll}
\boldsymbol{\varepsilon}_{B i}^{\mathrm{T}} & \boldsymbol{\xi}_{B i}^{\mathrm{T}} & \mathrm{K} & \boldsymbol{\varepsilon}_{A i}^{\mathrm{T}} & \boldsymbol{\xi}_{A i}^{\mathrm{T}} & \mathrm{K} & \boldsymbol{\varepsilon}_{C i}^{\mathrm{T}} & \boldsymbol{\xi}_{C i}^{\mathrm{T}}
\end{array}\right]^{\mathrm{T}} \\
\boldsymbol{f}_{i}==\left[\begin{array}{llllllll}
\boldsymbol{f}_{B i}^{\mathrm{T}} & \tau_{B i}^{\mathrm{T}} & \mathrm{K} & \boldsymbol{f}_{A i}^{\mathrm{T}} & \boldsymbol{\tau}_{A i}^{\mathrm{T}} & \mathrm{L} & \boldsymbol{f}_{C i}^{\mathrm{T}} & \boldsymbol{\tau}_{C i}^{\mathrm{T}}
\end{array}\right]^{\mathrm{T}}
\end{array}\right.
$$

where $\varepsilon_{B i}, \boldsymbol{\xi}_{B i}, \varepsilon_{A i}, \boldsymbol{\xi}_{A i}, \boldsymbol{\varepsilon}_{C i}$ and $\boldsymbol{\xi}_{C i}$ are linear and angular coordinates of nodes $B_{i}, A_{i}$ and $C_{i}$ in the frame $B_{i}-x_{i} y_{i} z_{i}$; $f_{B i}, \boldsymbol{\tau}_{B i}, \boldsymbol{f}_{A i}, \boldsymbol{\tau}_{A i}, \boldsymbol{f}_{C i}$ and $\boldsymbol{\tau}_{C i}$ are reaction forces and moments at $B_{i}, A_{i}$ and $C_{i}$ measured in $B_{i}-x_{i} y_{i} z_{i}$, respectively. The nodal coordinates can be related to $\boldsymbol{u}_{i}$ by

$$
\left\{\begin{array}{l}
\boldsymbol{\varepsilon}_{B i}=\boldsymbol{N}_{A i}^{B 1} \boldsymbol{u}_{i}, \boldsymbol{\xi}_{B i}=\boldsymbol{N}_{A i}^{B 2} \boldsymbol{u}_{i} \\
\boldsymbol{\varepsilon}_{A i}=\boldsymbol{N}_{A i}^{A 1} \boldsymbol{u}_{i}, \boldsymbol{\xi}_{A i}=\boldsymbol{N}_{A i}^{A 2} \boldsymbol{u}_{i}
\end{array}\right.
$$

where $\boldsymbol{N}_{A i}^{B 1}, \boldsymbol{N}_{A i}^{B 2}, \quad \boldsymbol{N}_{A i}^{A 1}$ and $\boldsymbol{N}_{A i}^{A 2}$ are transformation matrices of nodes $B_{i}$ and $A_{i}$ with respect to $\boldsymbol{u}_{i}$ in the frame $B_{i}-x_{i} y_{i} z_{i}$, respectively.

Thus, the coordinate transformation can be made to express Eq. (17) in the reference coordinate system $A-x y z$ as

$$
\boldsymbol{K}_{i} \boldsymbol{U}_{i}=\boldsymbol{F}_{i}
$$

where $\boldsymbol{K}_{i}=\boldsymbol{T}_{i} \boldsymbol{k}_{i} \boldsymbol{T}_{i}^{\mathrm{T}}, \boldsymbol{U}_{i}=\boldsymbol{T}_{i} \boldsymbol{u}_{i}, \boldsymbol{F}_{i}=\boldsymbol{T}_{i} \boldsymbol{f}_{i}$. Herein, $\boldsymbol{T}_{i}$ is the transformation matrix of the $i^{\text {th }}$ limb body fixed frame with respect to the global reference and we have

$$
\boldsymbol{T}_{i}=\operatorname{diag}\left[\boldsymbol{R}_{i} \cdots \boldsymbol{R}_{i}\right]
$$

where $\boldsymbol{R}_{i}$ is transformation matrix of $B_{i}-x_{i} y_{i} z_{i}$ with respect to $A-x y z$ and can be determined from the inverse kinematic analysis.

3.2. Equilibrium equation derivation of the moving platform

The free body diagram of the moving platform is shown in Fig. 6.

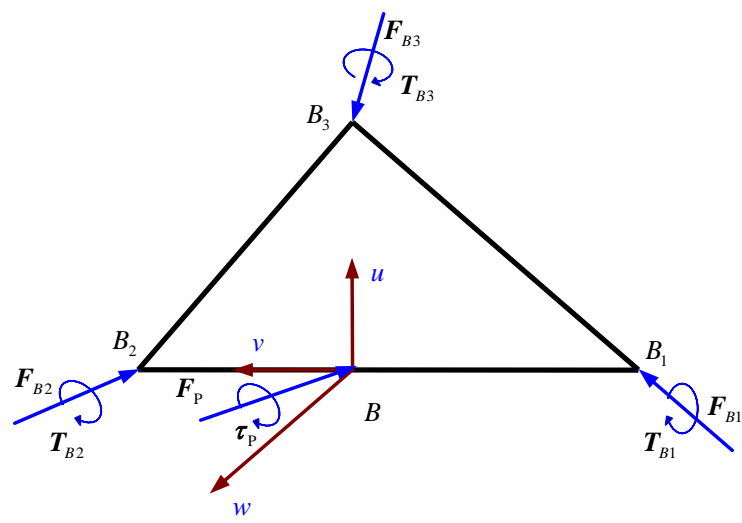

Fig. 6. Force diagram of the moving platform

According to Fig. 6, the static equations of the moving platform can be formulated

$$
\left\{\begin{array}{l}
-\sum_{i=1}^{3} \boldsymbol{F}_{B i}+\boldsymbol{F}_{\mathrm{P}}=\mathbf{0} \\
-\sum_{i=1}^{3} \boldsymbol{r}_{B i} \times \boldsymbol{F}_{B i}-\sum_{i=1}^{i=3} \boldsymbol{T}_{B i}+\tau_{\mathrm{P}}=\mathbf{0}
\end{array}\right.
$$

where $\boldsymbol{F}_{B i}$ and $\boldsymbol{T}_{B i}$ are the reaction force vectors at the interface between the moving platform and the $i^{\text {th }} \operatorname{limb}$ body measured in $A-x y z ; \boldsymbol{r}_{B i}$ is the vector pointing from point $B$ to $B_{i}$ measured in $A-x y z ; \boldsymbol{F}_{\mathrm{P}}$ and $\boldsymbol{\tau}_{\mathrm{P}}$ are the external force and moment acting on the moving platform (including the gravity of the moving platform), respectively. And there exist

$\boldsymbol{F}_{B i}=\boldsymbol{R}_{i} \boldsymbol{f}_{B i}, \quad \boldsymbol{T}_{B i}=\boldsymbol{R}_{i} \tau_{B i}$

\subsection{Deformation compatibility conditions}

As mentioned above, the moving platform connects with three limbs through three revolute joints, each of which can be treated as a virtual lump spring with equivalent stiffness. The displacement relationship between the platform and the limb can be demonstrated as Fig. 7, in which $B_{i \mathrm{M}}$ and $B_{i \mathrm{~L}}$ are the interface points associated with the moving platform and a limb, respectively. $\nabla \boldsymbol{B}_{i}$ and $\boldsymbol{\varepsilon}_{B i}$ are displacements of $B_{i \mathrm{M}}$ and $B_{i \mathrm{~L}}$ measured in the limb coordinate system $B_{i}-x_{i} y_{i} z_{i}$; $\boldsymbol{k}_{\mathrm{r} 1 i}=\operatorname{diag}\left[\begin{array}{lll}k_{\mathrm{r} x i} & k_{\mathrm{r} y i} & k_{\mathrm{r} z i}\end{array}\right]$ is the equivalent linear stiffness matrix of the $i^{\text {th }}$ revolute joint and $\boldsymbol{k}_{\mathrm{r} 2 i}=\operatorname{diag}\left[k_{\mathrm{r} u i} k_{\mathrm{r} v i} k_{\mathrm{r} w i}\right]$ is the equivalent angular stiffness matrix of the $i^{\text {th }}$ revolute joint. 


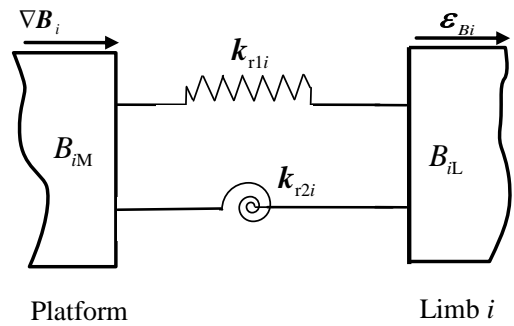

Fig. 7. Displacement relationship between the platform and the limb body

The elastic motion of the moving platform $\boldsymbol{U}_{\mathrm{P}}=\left[\begin{array}{ll}\varepsilon_{\mathrm{P}}^{\mathrm{T}} & \boldsymbol{\xi}_{\mathrm{P}}^{\mathrm{T}}\end{array}\right]^{\mathrm{T}}$ is caused by the deflections of three flexible limbs. The elastic displacement $\nabla \boldsymbol{B}_{i}$ of $B_{i \mathrm{M}}$ (fixed on the moving platform) can be derived as follows

$\nabla \boldsymbol{B}_{i}=\boldsymbol{R}_{i}^{\mathrm{T}} \boldsymbol{D}^{r i} \boldsymbol{U}_{\mathrm{p}}$

where $\boldsymbol{D}^{r_{i}}=\left[\begin{array}{ll}\mathbf{I}_{3 \times 3} & \hat{\boldsymbol{B}}_{i}\end{array}\right], \quad \hat{\boldsymbol{B}}_{i}$ is the skew-symmetric matrix of vector $\boldsymbol{b}_{i}$.

Thus the reaction forces of the revolute joint in the $i^{\text {th }}$ limb can be expressed as

$$
\left\{\begin{array}{l}
\boldsymbol{f}_{B i}=-\boldsymbol{k}_{\mathrm{r} 1 i}\left(\boldsymbol{N}_{A i}^{B 1} \boldsymbol{T}_{i}^{\mathrm{T}} \boldsymbol{U}_{i}-\boldsymbol{R}_{i}^{\mathrm{T}} \boldsymbol{D}^{r i} \boldsymbol{U}_{\mathrm{p}}\right) \\
\boldsymbol{\tau}_{B i}=-\boldsymbol{k}_{\mathrm{r} 2 i} \boldsymbol{N}_{A i}^{B 2} \boldsymbol{T}_{i}^{\mathrm{T}} \boldsymbol{U}_{i}
\end{array}\right.
$$

Similarly, the reactions of universal joint in the $i^{\text {th }}(i=1 \sim 2)$ limb can be expressed as

$$
\left\{\begin{array}{l}
\boldsymbol{f}_{A i}=-\boldsymbol{k}_{\mathrm{u} 1 i} \boldsymbol{N}_{A i}^{A 1} \boldsymbol{T}_{i}^{\mathrm{T}} \boldsymbol{U}_{i} \\
\boldsymbol{\tau}_{A i}=-\boldsymbol{k}_{\mathrm{u} 2 i} \boldsymbol{N}_{A i}^{A 2} \boldsymbol{T}_{i}^{\mathrm{T}} \boldsymbol{U}_{i}
\end{array}\right.
$$

where $\boldsymbol{k}_{\mathrm{u} 1 i}=\operatorname{diag}\left[k_{\mathrm{ux} i} k_{\mathrm{uy} i} k_{\mathrm{r} z i}\right]$ and $\boldsymbol{k}_{\mathrm{u} 2 i}=\operatorname{diag}\left[k_{\mathrm{u} u i} k_{\mathrm{u} v i} k_{\mathrm{r} w i}\right]$ are the equivalent stiffness of a universal joint in related directions.

The reactions of the spherical joint in limb 3 are

$$
\left\{\begin{array}{l}
\boldsymbol{f}_{A 3}=-\boldsymbol{k}_{\mathrm{s} 1} \boldsymbol{N}_{A 3}^{A 1} \boldsymbol{T}_{3}^{\mathrm{T}} \boldsymbol{U}_{3} \\
\tau_{A 3}=\mathbf{0}
\end{array}\right.
$$

where $\boldsymbol{k}_{\mathrm{s} 1}=\operatorname{diag}\left[\begin{array}{lll}k_{\mathrm{s} x} & k_{\mathrm{s} y} & k_{\mathrm{sz}}\end{array}\right]$ is the linear stiffness of the spherical joint in limb 3 .

\subsection{Governing equilibrium equation of the system}

Substituting Eqs. (25) (27) into Eqs. (20) and (22), one can write the equilibrium equations of the Exechon $\mathrm{PKM}$ as

$R Q^{\circ}=$ Fo where $\tilde{\boldsymbol{K}}$ is the global stiffness matrix; $\tilde{\boldsymbol{U}}$ and $\tilde{\boldsymbol{F}}$ are the general coordinates and external loads vectors. And there exist

$$
\boldsymbol{K}^{\circ}=\left[\begin{array}{llll}
\boldsymbol{K}_{1,1} & & & \boldsymbol{K}_{1,4} \\
& \boldsymbol{K}_{2,2} & & \boldsymbol{K}_{2,4} \\
& & \boldsymbol{K}_{3,3} & \boldsymbol{K}_{3,4} \\
\boldsymbol{K}_{4,1} & \boldsymbol{K}_{4,2} & \boldsymbol{K}_{4,3} & \boldsymbol{K}_{4,4}
\end{array}\right]
$$

$\boldsymbol{K}_{i, i}=\left[\begin{array}{c}\boldsymbol{R}_{i} \boldsymbol{k}_{\mathrm{r} 1 i} \boldsymbol{N}_{A i}^{B 1} \boldsymbol{T}_{i}^{\mathrm{T}} \\ \boldsymbol{R}_{i} \boldsymbol{k}_{\mathrm{r} 2 i} \boldsymbol{N}_{A i}^{B 2} \boldsymbol{T}_{i}^{\mathrm{T}} \\ \mathrm{M} \\ \boldsymbol{R}_{i} \boldsymbol{k}_{\mathrm{u} 1 i} \boldsymbol{N}_{A i}^{A 1} \boldsymbol{T}_{i}^{\mathrm{T}} \\ \boldsymbol{R}_{i} \boldsymbol{k}_{\mathrm{u} 2 i} \boldsymbol{N}_{A i}^{A 2} \boldsymbol{T}_{i}^{\mathrm{T}} \\ \mathrm{M} \\ \mathbf{0} \\ \mathbf{0}\end{array}\right]+\boldsymbol{K}_{i} \quad(i=1,2)$

$$
\boldsymbol{K}_{3,3}=\left[\begin{array}{c}
\boldsymbol{R}_{3} \boldsymbol{k}_{\mathrm{r} 13} \boldsymbol{N}_{A 3}^{B 1} \boldsymbol{T}_{3}^{\mathrm{T}} \\
\boldsymbol{R}_{3} \boldsymbol{k}_{\mathrm{r} 23} \boldsymbol{N}_{A 3}^{B 2} \boldsymbol{T}_{3}^{\mathrm{T}} \\
\mathrm{M} \\
\boldsymbol{R}_{3} \boldsymbol{k}_{\mathrm{s} 1} \boldsymbol{N}_{A 3}^{A 1} \boldsymbol{T}_{3}^{\mathrm{T}} \\
\mathbf{0} \\
\mathrm{M} \\
\mathbf{0} \\
\mathbf{0}
\end{array}\right]+\boldsymbol{K}_{3}
$$

$$
\boldsymbol{K}_{4,4}=\left[\begin{array}{c}
\sum_{i=1}^{3} \boldsymbol{R}_{i} \boldsymbol{k}_{\mathrm{r} 1 i} \boldsymbol{R}_{i}^{\mathrm{T}} \boldsymbol{D}^{r_{i}} \\
\sum_{i=1}^{3} \boldsymbol{r}_{B i} \times \boldsymbol{R}_{i} \boldsymbol{k}_{\mathrm{r} 1 i} \boldsymbol{R}_{i}^{\mathrm{T}} \boldsymbol{D}^{r_{i}}
\end{array}\right]
$$

$$
\boldsymbol{K}_{4, i}=\left[\begin{array}{c}
-\boldsymbol{R}_{i} \boldsymbol{k}_{\mathrm{r} 1 i} \boldsymbol{N}_{A i}^{B 1} \boldsymbol{T}_{i}^{\mathrm{T}} \\
-\boldsymbol{r}_{B i} \times \boldsymbol{R}_{i} \boldsymbol{k}_{\mathrm{r} 1 i} \boldsymbol{N}_{A i}^{B 1} \boldsymbol{T}_{i}^{\mathrm{T}}-\boldsymbol{R}_{i} \boldsymbol{k}_{\mathrm{r} 2 i} \boldsymbol{N}_{A i}^{B 2} \boldsymbol{T}_{i}^{\mathrm{T}}
\end{array}\right]
$$

$\boldsymbol{K}_{i, 4}=\left[\begin{array}{c}-\boldsymbol{R}_{i} \boldsymbol{k}_{\mathrm{r} 1 i} \boldsymbol{R}_{i}^{\mathrm{T}} \boldsymbol{D}^{r_{i}} \\ \mathbf{0}\end{array}\right]$

3.5. Formulation of stiffness matrix of the moving platform

Eq. (29) gives the formulation of the global stiffness matrix, from which it can be found that it is non-diagonal, indicating that the stiffness of the moving platform is coupled with those of the limb structures. In order to evaluate the rigidity of the moving platform, the concept 
of compliance is adopted, which is mathematically expressed as

$$
\mathcal{C}^{\circ}=\left[\mathrm{R}^{-1}\right]_{H \times H}
$$

where $H=18 n+6$ is the dimension of the stiffness matrix, $n$ is the discrete nodes number of each limb.

With Eq. (35), the compliance matrix of the platform can be obtained as the last $6 \times 6$ block matrix in $\mathcal{E}^{\circ}$ and is denoted as $\boldsymbol{C}_{\mathrm{p}}$. Therefore, the stiffness of the platform in the body fixed frame $B-u v w$ can be expressed according to the duality of compliance as

$$
\boldsymbol{K}_{\mathrm{p}}=\boldsymbol{T}_{0}^{\mathrm{T}} \boldsymbol{C}_{\mathrm{p}}^{-1} \boldsymbol{T}_{0}=\boldsymbol{T}_{0}^{\mathrm{T}}\left[\left.\boldsymbol{R}^{-1}\right|_{(H-18 n) \times(H-18 n)}\right]^{-1} \boldsymbol{T}_{0}
$$

where $\boldsymbol{T}_{0}=\operatorname{diag}\left[\boldsymbol{R}_{0} \boldsymbol{R}_{0}\right]$ is the transformation matrix of the platform body fixed frame with respect to the global reference.

It is noted that the overall stiffness of the Exechon machine is greatly influenced by its hand axes. Compared to the parallel part, the hand axes are quite soft due to its unique serial structures. As a result, the comparatively softer serial part has detrimental effects on machining properties with no doubt. However, it is worth noting that in the present study the compliance of serial hand axes is not included subject to the following reason. The basic consideration is that the serial part of an Exechon-type machine could be in different forms when designed to achieve various tasks such as milling, drilling, riveting, etc. In other words, the serial parts can be regarded as a reconfigurable plug-and-play unit with different functions and structures. As a result, the stiffness of this serial part can only be determined according to its specific structures. On the contrary, the parallel kinematic part of an Exechon-type machine possesses a common architecture and can be regarded as a general flexible supporting structure for the serial part. With this thought, the overall stiffness of the hybrid machine can be calculated through the superposition of the serial hand axes stiffness $\boldsymbol{K}_{\mathrm{m}}$ and the parallel platform stiffness $\boldsymbol{K}_{\mathrm{p}}$ with kinematic transformations.

\section{Stiffness Analysis}

\subsection{Parameters of an exemplar system}

The major geometrical parameters of an example system of the Exechon module are listed in Table 1. Herein, $s$ denotes the stroke of the moving platform; $\psi_{\max }$ and $\theta_{\max }$ denote the maximum rotation angle of precession and nutation respectively; $d_{\max }$ and $d_{\min }$ represent the maximum and minimum distances between $A_{i}$ and $B_{i}$; the meaning of the other symbols can be referred to aforementioned context.

Table 2 gives the stiffness coefficients of three perpendicular axes of joints in their local frames, which are calculated through ANSYS software.

Table 1 The geometrical parameters of the Exechon PKM

\begin{tabular}{cccccccc}
\hline$r_{\mathrm{P}}$ & $r_{\mathrm{b}}$ & $s$ & $d_{\min }$ & $d_{\max }$ & $\psi_{\max }$ & $\theta_{\max }$ & $l_{n}$ \\
$\mathrm{~mm}$ & $\mathrm{~mm}$ & $\mathrm{~mm}$ & $\mathrm{~mm}$ & $\mathrm{~mm}$ & $\left(^{\circ}\right.$ & $\left(^{\circ}\right.$ & $\mathrm{mm}$ \\
\hline 220 & 600 & 550 & 736 & 2600 & \pm 40 & \pm 30 & 188.5 \\
\hline
\end{tabular}

Table 2 The stiffness coefficients of joints in local frames (unit: $\mathrm{N} / \mathrm{um}, 10^{6} \mathrm{~N} \cdot \mathrm{m} / \mathrm{rad}$ )

\begin{tabular}{ccccccccc}
\hline$k_{\mathrm{ux}}$ & $k_{\mathrm{uy}}$ & $k_{\mathrm{u} z}$ & $k_{\mathrm{u} w}$ & $k_{\mathrm{s} x}$ & $k_{\mathrm{s} y}$ & $k_{\mathrm{s} z}$ & $k_{\mathrm{r} 1 x}$ & $k_{\mathrm{r} 1 y}$ \\
\hline 380 & 530 & 1006 & 18 & 380 & 530 & 530 & 23 & 23 \\
$k_{\mathrm{r} 1 z}$ & $k_{\mathrm{r} 1 v}$ & $k_{\mathrm{r} 1 w}$ & $k_{\mathrm{r} 2 x}$ & $k_{\mathrm{r} 2 y}$ & $k_{\mathrm{r} 2 z}$ & $k_{\mathrm{r} 2 u}$ & $k_{\mathrm{r} 2 v}$ & $k_{\mathrm{r} 2 w}$ \\
623 & 18 & 18 & 112 & 214 & 100 & 24 & 20 & 20 \\
\hline
\end{tabular}

According to the parameters above of the example system, the following numerical simulation and analysis can be conducted.

\subsection{Stiffness matrix at extreme position}

By solving Eq. (36), one can easily obtain the stiffness of the platform at any given configuration. Taking the Exechon PKM at the extreme position for example, where $p_{z}=1.3 \mathrm{~m}, \theta=0^{\circ}, \psi=0^{\circ}$, the results are listed in Eq. (37).

$$
\boldsymbol{K}_{\mathrm{P}}=\left[\begin{array}{llllll}
k_{11} & k_{12} & k_{13} & k_{14} & k_{15} & k_{16} \\
k_{21} & k_{22} & k_{23} & k_{24} & k_{25} & k_{26} \\
k_{31} & k_{32} & k_{33} & k_{34} & k_{35} & k_{36} \\
k_{41} & k_{42} & k_{43} & k_{44} & k_{45} & k_{46} \\
k_{51} & k_{52} & k_{53} & k_{54} & k_{55} & k_{56} \\
k_{61} & k_{62} & k_{63} & k_{64} & k_{65} & k_{66}
\end{array}\right]=\left[\begin{array}{cccccc}
12.1618 & 0 & -18.9769 & 0 & 19.9853 & 0 \\
0 & 15.1464 & 0 & -28.6007 & 0 & 0.7276 \\
-18.9769 & 0 & 203.4800 & 0 & -38.9526 & 0 \\
0 & -28.6007 & 0 & 49.9638 & 0 & -0.0140 \\
19.9853 & 0 & -38.9526 & 0 & 23.5366 & 0 \\
0 & 0.7276 & 0 & -0.0140 & 0 & 1.2975
\end{array}\right] \times 10^{6}
$$


From the perspective of physics, the stiffness matrix denotes the ability of the moving platform to resist deflection subject to external loads. For the sake of clarity, the diagonal elements in the matrix are defined as principle stiffnesses while the non-diagonal elements as coupled stiffnesses. For content limitation, only the linear principle stiffnesses and the angular principle stiffnesses are analyzed.

As can be seen from the matrix, the linear principle stiffnesses along $u$ and $v$ axes are much less than that along $w$ axis. This implies that the PKM claims the highest rigidity performance along the $w$ direction of the platform. On the contrary, the angular principle stiffnesses about $u$ and $v$ axes are much higher than that about $w$ axis, indicating a 'weakest' rigidity about the $w$ direction of the platform.

In order to validate the accuracy of the proposed model, a numerical stiffness model for the Exechon PKM module shown in Fig. 1 is developed in ANSYS Workbench to evaluate the stiffness properties at a typical configuration where $p_{z}=1.075 \mathrm{~m}, \theta=0^{\circ}, \psi=0^{\circ}$. For this purpose, a $1000 \mathrm{~N}$ force is applied at the center of the platform along $u, v$ and $w$ axes, respectively. Then a static analysis is conducted, leading to corresponding deformations of the PKM as demonstrated in Fig. 8. The obtained analytical stiffness results are listed in Eq. (38).
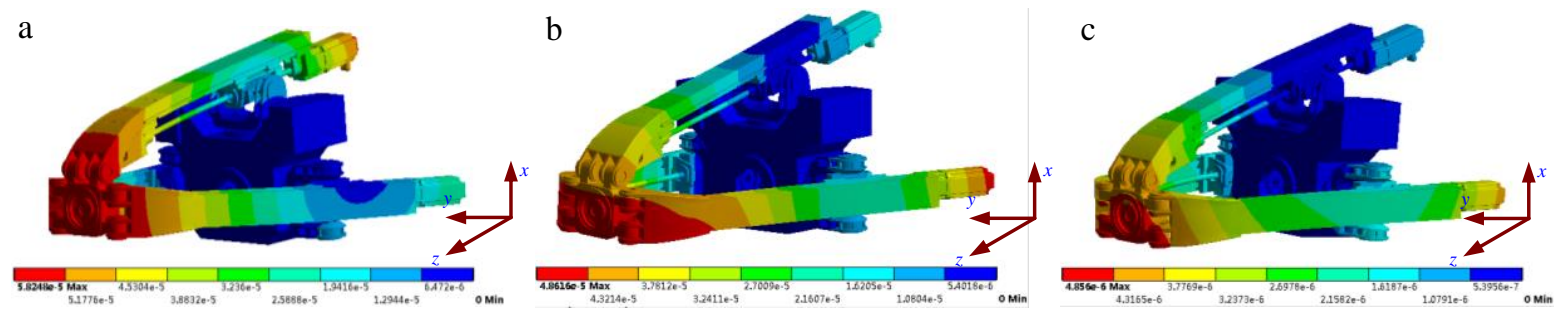

Fig. 8. Deformations of the Exechon PKM: (a) in $u$ direction, (b) in $v$ direction, and (c) in $w$ direction.

$$
\boldsymbol{K}_{\mathrm{P}}=\left[\begin{array}{llllll}
k_{11} & k_{12} & k_{13} & k_{14} & k_{15} & k_{16} \\
k_{21} & k_{22} & k_{23} & k_{24} & k_{25} & k_{26} \\
k_{31} & k_{32} & k_{33} & k_{34} & k_{35} & k_{36} \\
k_{41} & k_{42} & k_{43} & k_{44} & k_{45} & k_{46} \\
k_{51} & k_{52} & k_{53} & k_{54} & k_{55} & k_{56} \\
k_{61} & k_{62} & k_{63} & k_{64} & k_{65} & k_{66}
\end{array}\right]=\left[\begin{array}{cccccc}
16.9936 & 0 & -22.3108 & 0 & 23.1765 & 0 \\
0 & 21.3942 & 0 & -33.4799 & 0 & 1.0018 \\
-22.3108 & 0 & 197.8913 & 0 & -37.8696 & 0 \\
0 & -33.4799 & 0 & 48.5159 & 0 & 0 \\
23.1765 & 0 & -37.8696 & 0 & 22.7218 & 0 \\
0 & 1.0018 & 0 & 0 & 0 & 1.8032
\end{array}\right] \times 10^{6}
$$

As can be clearly seen from Fig. 8, the deformations of the limb bodies and the joints are quite large thus can not be ignored and should be considered during stiffness estimation. On the contrary, the elastic deformations of the base and the platform are comparatively smaller, verifying the assumption of rigid base and platform is reasonable. With force-deflection relationships along the three perpendicular projections, the stiffness values of the platform are obtained and compared with analytical results as listed in Table 3.

Table 3 Comparison of analytic and numerical results

\begin{tabular}{lccc}
\hline & $k_{11}$ & $k_{22}$ & $k_{33}$ \\
\hline Analytic results (N/um) & 16.994 & 21.394 & 197.891 \\
FEA simulations (N/um) & 17.168 & 20.596 & 205.931 \\
Calculation errors & $1.02 \%$ & $4.01 \%$ & $3.90 \%$ \\
\hline
\end{tabular}

As can be seen, errors between the analytical results and the FEA simulations are less than 5\%. This indicates that the assumed analysis can follow the actual design, thus can be applied to the stiffness evaluation of the Exechon PKM.

\subsection{Stiffness distributions over workspace}

A numerical approach is applied to evaluate the PKM's stiffness properties throughout the workspace. In the numerical simulation, the workspace is partitioned into a finite number of elements. Each element is represented by the coordinates of its centre point, and its stiffness is calculated accordingly. For the sake of generality, the following illustrates the distributions of the six principle stiffnesses over the work plane at $p_{z}=1.3$ $\mathrm{m}$ as shown in Fig. 9.

From Fig. 9, it can be easily observed that the six principle stiffnesses are axisymmetric over the given work plane. This is coincident with the structural features that limb 1 and limb 2 are symmetrical with respect to 
limb 3. It also indicates that the stiffness properties of the PKM are strongly dependent on the mechanism's configurations. For example, the linear principle stiffness along $u$ axis $k_{11}$ varies from the minimal value of $3.74 \times 10^{6}$ to the maximal value of $1.25 \times 10^{7}$, and the linear principle stiffness along $v$ axis $k_{22}$ changes from $3.01 \times 10^{6}$ to $1.53 \times 10^{7}$. The linear principle stiffness along $w$ axis $k_{33}$ varies from $2.03 \times 10^{8}$ to $2.12 \times 10^{8}$.
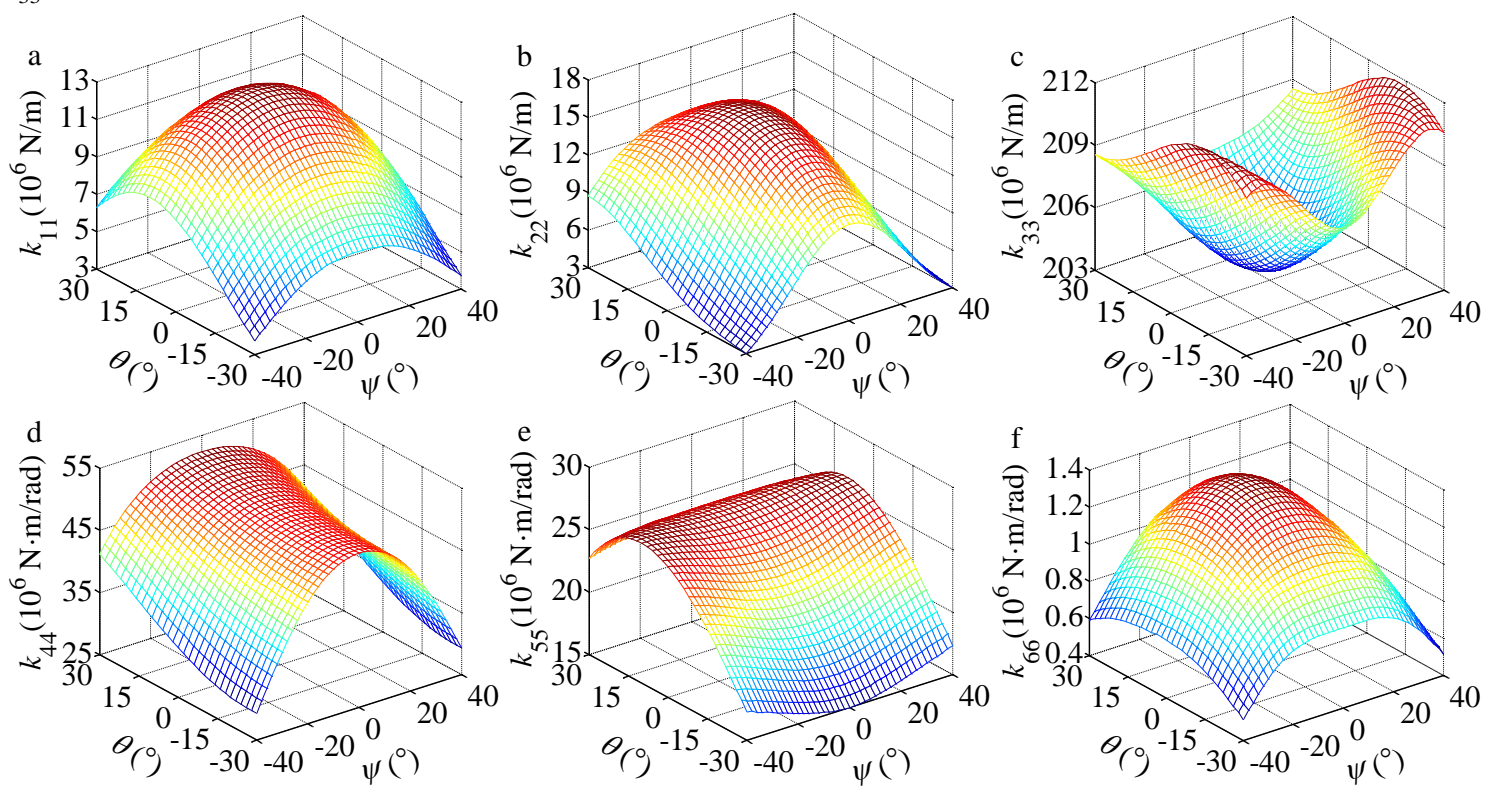

Fig. 9. Stiffness distributions over work plane of $p_{z}=1.3 \mathrm{~m}$ : (a) linear stiffness along $u$ axis, (b) linear stiffness along $v$ axis, (c) linear stiffness along $w$ axis, (d) angular stiffness about $u$ axis, (e) angular stiffness about $v$ axis, and (f) angular stiffness about $w$ axis.

Besides the two rotational capacities about the $x / y$ axes, the Exechon PKM also possesses a translational capacity along the $z$ axis. The following depicts the variations of the six principle stiffnesses with respect to the change of $p_{z}$, i.e., the central distance between the platform and the base, in which $p_{z}$ is set to vary from $0.75 \mathrm{~m}$ to $1.3 \mathrm{~m}$. The precession angle and nutation angle keep unchanged. For the sake of generality, $\theta=0^{\circ}, \psi=0^{\circ}$.
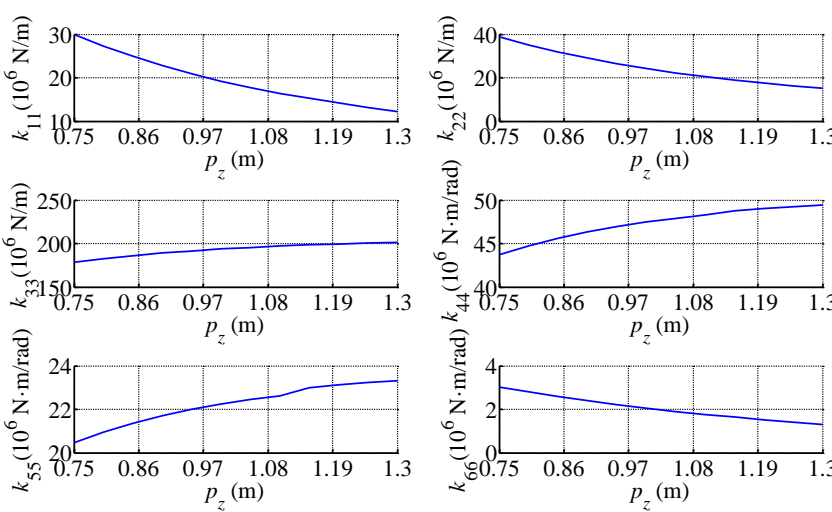

Fig. 10. Variations of principle stiffness with respect to $p_{\mathrm{z}}$

It can be observed from Fig. 10 that the principle stiffnesses $k_{11}, k_{22}$ and $k_{66}$ decrease monotonously to the increment of $p_{z}$ while the other three principle stiffnesses increase monotonously. The decreasing stiffnesses along $u / v$ axes and about $w$ axis is obvious if only the limbs are regarded as cantilevers. The reason for the increasing stiffnesses along $w$ axis and about $u / v$ axes may lie in that a larger central distance between the platform and the base means a smaller tilting angle of the limbs.

\subsection{Parametric analysis}

In this subsection, the effects of some design variables on the stiffness properties of the PKM are investigated. Some structural parameters, dimensional parameters and stiffness parameters are taken as design variables and their influences on system stiffness analyzed as follows.

\subsubsection{Structural parameter effects}

Fig. 11 shows the variations of the six principle stiffnesses with respect to the changes of limb body cross-section, in which the equivalent width $w_{1}$ changes from $0.08 \mathrm{~m}$ to $0.18 \mathrm{~m}$ and the equivalent height $h_{1}$ changes from $0.2 \mathrm{~m}$ to $0.3 \mathrm{~m}$. 
From Fig. 11, it can be easily found that the six principle stiffnesses increase monotonously with the increment of limb body cross-section. This is obviously consistent with the physical fact that a larger cross-section means a higher rigidity of the limb body and thus 'stiffens' the PKM.
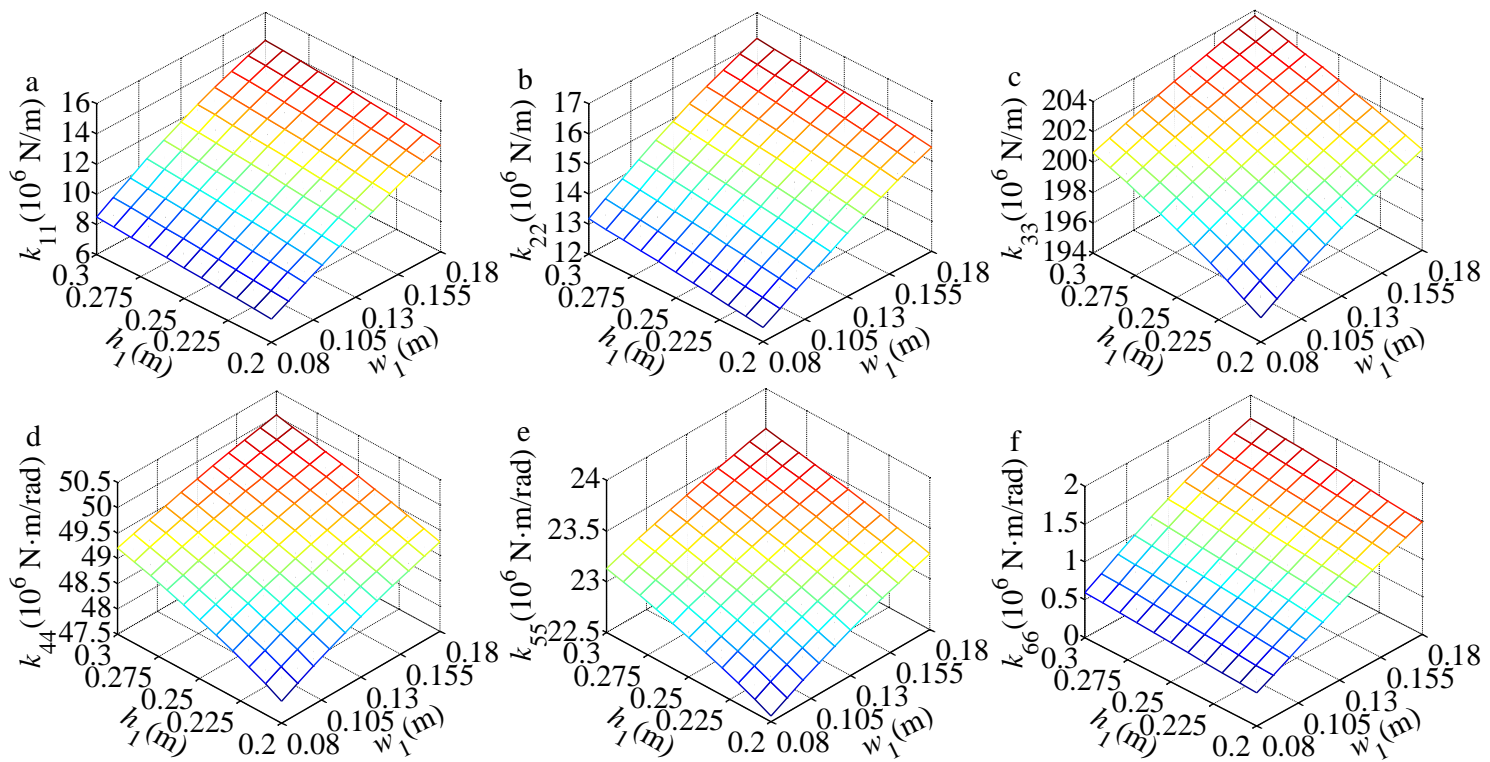

Fig. 11. Variations of principle stiffness with respect to $w_{1}$ and $h_{1}$ : (a) linear stiffness along $u$ axis, (b) linear stiffness along $v$ axis, (c) linear stiffness along $w$ axis, (d) angular stiffness about $u$ axis, (e) angular stiffness about $v$ axis, and (f) angular stiffness about $w$ axis.
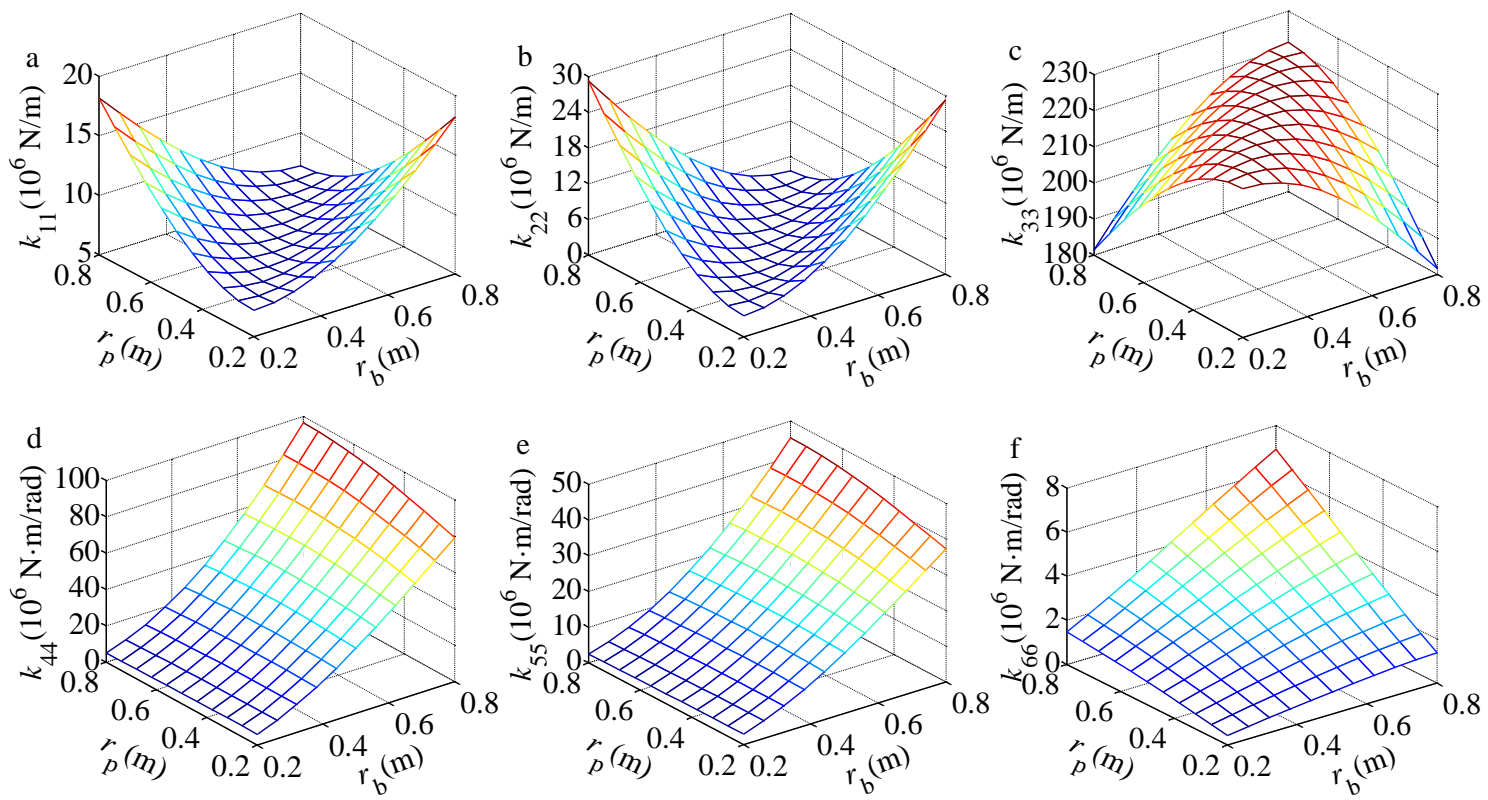

Fig. 12. Variations of principle stiffnesses with respect to $r_{\mathrm{p}}$ and $r_{\mathrm{b}}$ : (a) linear stiffness along $u$ axis, (b) linear stiffness along $v$ axis, (c) linear stiffness along $w$ axis, (d) angular stiffness about $u$ axis, (e) angular stiffness about $v$ axis, and (f) angular stiffness about $w$ axis.

\subsubsection{Dimensional parameter effects}

Fig. 12 shows the variations of the six principle stiffnesses with respect to the changes of the radii of the platform and the base, in which the radius of the platform $r_{\mathrm{p}}$ changes from $0.2 \mathrm{~m}$ to $0.8 \mathrm{~m}$ and the radius of the base $r_{\mathrm{b}}$ changes from $0.2 \mathrm{~m}$ to $0.8 \mathrm{~m}$.
As can be seen clearly from Fig. 12, the variation curves of $k_{11}, k_{22}$ and $k_{33}$ are symmetric about the line of $r_{\mathrm{p}}=r_{\mathrm{b}}$, which means that the two dimensional parameters of $r_{\mathrm{p}}$ and $r_{\mathrm{b}}$ have the same 'intensity' on the linear stiffnesses. About this symmetry axis, the effects of dimensional parameters on the three principle stiffnesses 
are different in that $k_{11}$ and $k_{22}$ increase monotonously with the increment of $r_{\mathrm{p}}$ and $r_{\mathrm{b}}$ while $k_{33}$ decreases monotonously with the increment of $r_{\mathrm{p}}$ and $r_{\mathrm{b}}$. With this observation, it might be considerable to take a strategy of $r_{\mathrm{p}}=r_{\mathrm{b}}$ during the conceptual design stage for this kind of PKM module. The three angular stiffnesses $k_{44}, k_{55}$ and $k_{66}$ increase monotonously with the increment of $r_{\mathrm{p}}$ and $r_{\mathrm{b}}$. The variation curves of the three angular principle stiffnesses are different in that the base radius $r_{\mathrm{b}}$ has a 'stronger' impact on $k_{44}$ and $k_{55}$ while almost having the same intensity as $r_{\mathrm{p}}$ on $k_{66}$. This phenomenon can be explained like that when the radius of the base $r_{\mathrm{b}}$ is constant, the bigger the radius of the platform $r_{\mathrm{p}}$ becomes, the easier the platform rotates about its axis.

\subsubsection{Stiffness parameter effects}

The following demonstrates the variations of the six principle stiffnesses with respect to the stiffness coefficients of the revolute joint, the universal joint and the spherical joint.

For the convenience of analysis, some physical quantities are defined. For example, define

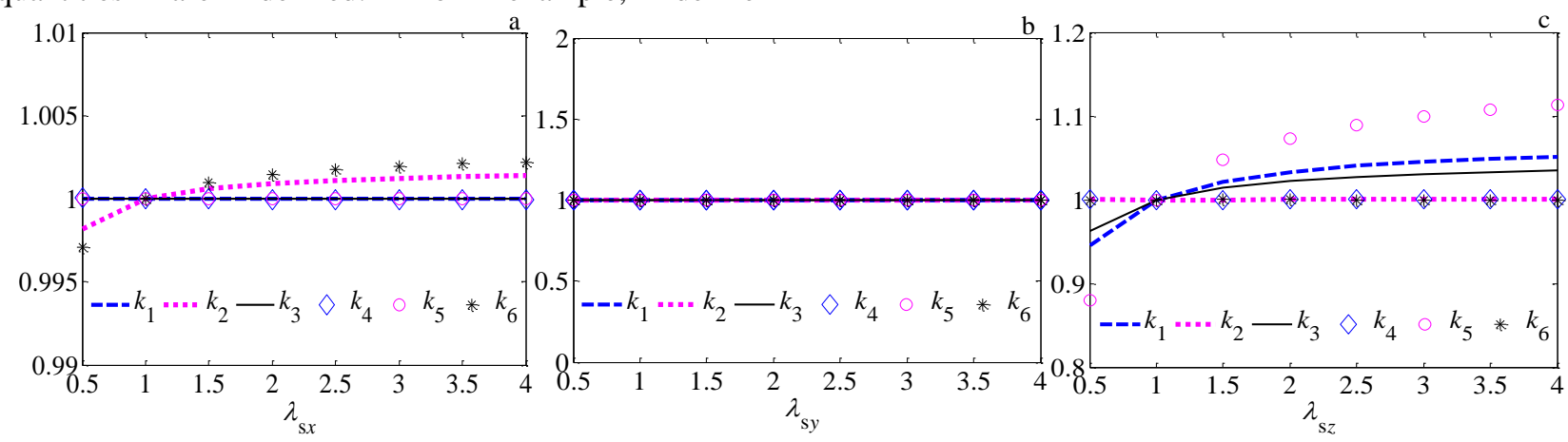

Fig. 13. Variations of $k_{i}$ with respect to $k_{\mathrm{s}}$ : (a) variations of $k_{i}$ with respect to $\lambda_{\mathrm{s} x}$, (b) variations of $k_{i}$ with respect to $\lambda_{\mathrm{s} y}$, and (c) variations of $k_{i}$ with respect to $\lambda_{\mathrm{s} z}$.

Fig. 14 shows the variations of $k_{i}$ with respect to three linear stiffnesses $k_{\mathrm{u} x}, k_{\mathrm{u} y}$ and $k_{\mathrm{uz}}$ along three perpendicular axes and angular stiffness $k_{\mathrm{u} w}$ about the $z$ axis of a universal joint. It is clear that $\lambda_{\mathrm{u} y}$ and $\lambda_{\mathrm{uw}}$ have no influence on $k_{i}$ indicating that the linear stiffness along the $z$ axis $k_{\mathrm{u} z}$ and the angular stiffness about the $z$ axis $k_{\mathrm{u} w}$ have no influence on the six principle stiffnesses. Comparing Fig. 14(a) with Fig. 14(c), it can be found that $k_{1}$ and $k_{6}$ increase monotonously with the increment of $\lambda_{\mathrm{ux}}$ while $k_{2}, k_{3}$ and $k_{4}$ increase with the increment of $\lambda_{\mathrm{uz} z}$. The linear stiffnesses along $x$ and $z$ directions should be paid more attention to rather than the angular stiffness in the universal joint.

Fig. 15 shows the variations of $k_{i}$ with respect to the stiffness of the revolute joint. As shown in Fig. 3, the non-dimensional factor $k_{i}$ as $k_{i}=k_{i i} / k_{i i 0}$ in which $k_{i i 0}$ is the principle stiffness of the example system in the corresponding directions shown in Eq. (37). Assume $\lambda_{\mathrm{s} x}$ is the amplification factor of $k_{\mathrm{s} x}$ shown in Table 2. In a similar way, $\lambda_{\mathrm{sy}}, \lambda_{\mathrm{sz}}, \lambda_{\mathrm{ux}}, \lambda_{\mathrm{uy}}, \lambda_{\mathrm{uz} z}, \lambda_{\mathrm{uw}}, \lambda_{\mathrm{r} 1 x}, \lambda_{\mathrm{r} 1 y}, \lambda_{\mathrm{r} 1 z}, \lambda_{\mathrm{r} 1 v}, \lambda_{\mathrm{r} 1 w}$, $\lambda_{\mathrm{r} 2 x}, \lambda_{\mathrm{r} 2 y}, \lambda_{\mathrm{r} 2 z}, \lambda_{\mathrm{r} 2 u}, \lambda_{\mathrm{r} 2 v}$ and $\lambda_{\mathrm{r} 2 w}$ are defined.

Fig. 13 shows the variations of $k_{i}$ with respect to the stiffness of the spherical joint. It can be observed that $k_{i}$ keeps unchanged with respect to $\lambda_{\text {sy }}$ indicating that the stiffness in $y$ direction of the spherical joint $k_{\text {sy }}$ has no influence on the six principle stiffnesses. $k_{2}$ and $k_{6}$ increase with the increment of $\lambda_{\mathrm{s} x}$ and the increasing tendency becomes slowly when $\lambda_{\mathrm{s} x} \geq 3$, indicating that $k_{\mathrm{s} x}$ only affects the linear stiffness $k_{22}$ and the angular stiffness $k_{66}$ in a small scale. Similarly, $k_{1}, k_{3}$ and $k_{5}$ increase with the increment of $\lambda_{\mathrm{s} z}$ while the others keep unchanged. Therefore, it is considerable to pay more attention to the linear stiffness of a spherical joint in $z$ axis. revolute joint is consist of Part 1 and Part 2. Assume $\lambda_{\mathrm{r} 1 x}$, $\lambda_{\mathrm{r} 1 y} \lambda_{\mathrm{r} 1 z}$ and $\lambda_{\mathrm{r} 1 v}, \lambda_{\mathrm{r} 1 w}$ denote amplification factors of linear stiffnesses and angular stiffnesses along and about three perpendicular axes in Part 1 of the revolute joint while $\lambda_{\mathrm{r} 2 x}, \lambda_{\mathrm{r} 2 y} \lambda_{\mathrm{r} 2 z}$ and $\lambda_{\mathrm{r} 2 u}, \lambda_{\mathrm{r} 2 v}$ and $\lambda_{\mathrm{r} 2 w}$ are those of Part 2 of the revolute joint. As shown in Fig. 15, the stiffness of two parts of the revolute joint have the same intensity on the principle stiffnesses of the platform. Obviously, the stiffnesses about the $x$ and $z$ axes have no effect on the stiffness of the platform. And $k_{1}, k_{2}$ and $k_{6}$ increase monotonously with the increment of $\lambda_{\mathrm{r} 1 x}, \lambda_{\mathrm{r} 2 x}, \lambda_{\mathrm{r} 1 v}$ and $\lambda_{\mathrm{r} 2 v}$. $k_{6}$ keeps unchanged while the others increase monotonously with the increment of $\lambda_{\mathrm{r} 1 z}$ and $\lambda_{\mathrm{r} 2 z}$. Compared the effects of the linear stiffness along the $y$ linear stiffness along the $y$ axis and the angular 
axis and that along the $z$ axis, it seems that stiffness along the $z$ axis has a 'stronger' impact on the principle
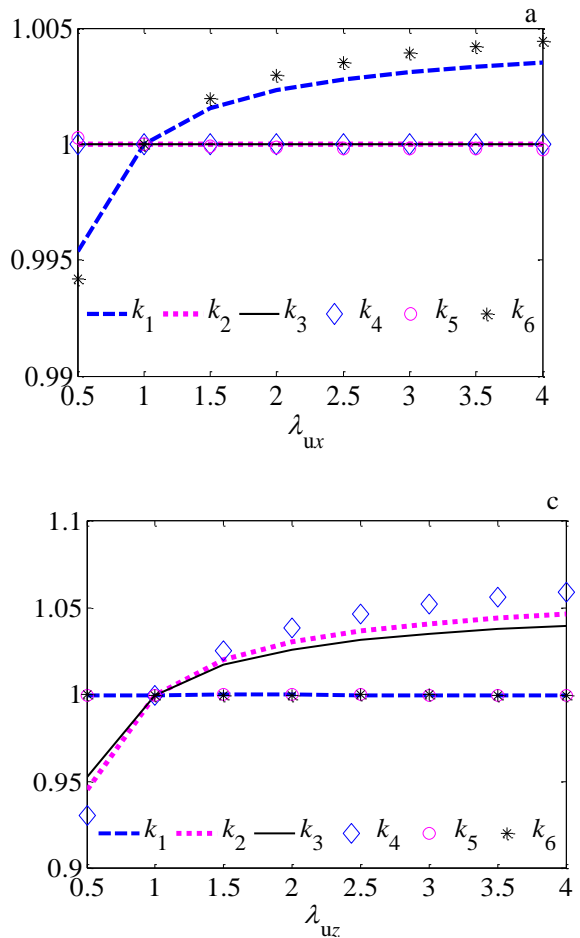

stiffness.
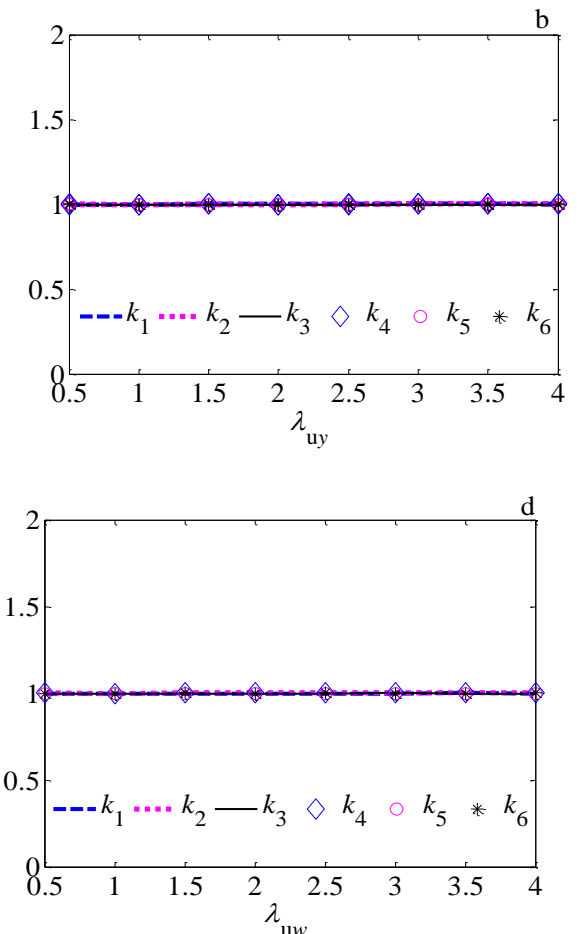

Fig. 14. Variations of $k_{i}$ with respect to $k_{\mathrm{u}}$ : (a) variations of $k_{i}$ with respect to $\lambda_{\mathrm{ux}}$, (b) variations of $k_{i}$ with respect to $\lambda_{\mathrm{uy}}$, (c) variations of $k_{i}$ with respect to $\lambda_{\mathrm{uz}}$, and (d) variations of $k_{i}$ with respect to $\lambda_{\mathrm{uw}}$.

With the analysis on Figs. 13 15, it is suggested that more attention should be paid to the linear stiffnesses along $x$ and $z$ axes as well as the angular stiffness about $y$ axis when design such a PKM.

\section{Conclusions}

This paper has produced an in-depth study on the stiffness characteristics of the Exechon module through the kinetostatics methodology. The stiffness model proposed in this paper considers the deflections of the limb structure, the revolute joint assembly, the spherical joint and the universal joint. Compared with finite element models, the analytical model proposed in this paper is much more succinct and can be numerically solved with high computational efficiency. The major conclusions and contributions of this study can be summarised as the followings:

(1) An analytical kinetostatic model for the stiffness analysis of Exechon PKM is established with the technique of substructure synthesis, in which the limb assemblage flexibility is accounted through FE formulation by treating it as a spatial beam with a uniform cross-section supported by two sets of lumped virtual springs of joints. The proposed methodology of stiffness modelling is also applicable to other kinds of PKMs.

(2) Based on the proposed kinetostatic model, the stiffness matrix of the platform is formulated, and the distributions of six principle stiffnesses are predicted to demonstrate a strong position-dependency and symmetry relative to work plane aroused by the symmetric structural features. Compared with traditional FE method and virtual joint method, the present method for stiffness evaluation is much more concise and effective.

(3) Parametric effects including structural, dimensional and stiffness parameters on the PKM's rigidity properties are explored to offer significant guidances for the early design stage of such a PKM module.

Readers should note that two limitations of the proposed model are: (1) the cross-section of the limb is assumed to be constant. This assumption should be modified in our future study so that the model proposed can be more accurate. (2) the kinetostatic model proposed can be easily extended to a elastodynamic model thus can be used to evaluate the dynamic stiffness and steady responses which is currently under research by the authors and the in-depth analysis will be presented elsewhere. 


\section{Acknowledgments}

This work was supported by Open Research Fund of Key Laboratory of High Performance Complex Manufacturing, Central South University with Grant No. Kfkt2013-12 and Open Fund of Shanghai Key Laboratory of Digital Manufacture for Thin-walled Structures with Grant No. 2014002.
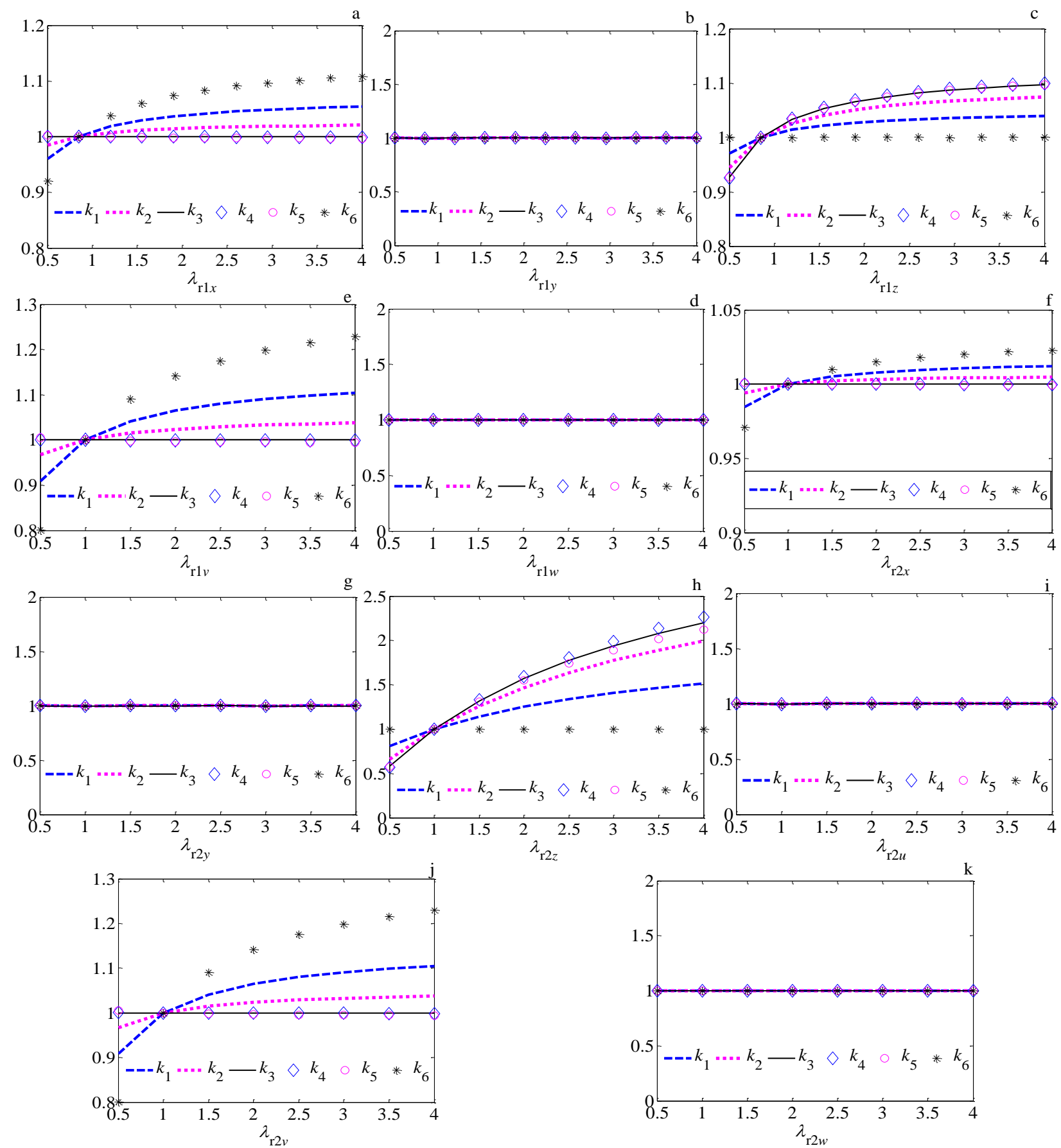

Fig. 15. Variations of $k_{i}$ with respect to $k_{\mathrm{r}}$ : (a) variations of $k_{i}$ with respect to $\lambda_{\mathrm{r} 1 x}$, (b) variations of $k_{i}$ with respect to $\lambda_{\mathrm{r} 1 y}$, (c) variations of $k_{i}$ with respect to $\lambda_{\mathrm{r} 1 z}$, (d) variations of $k_{i}$ with respect to $\lambda_{\mathrm{r} 1 v}$, (e) variations of $k_{i}$ with respect to $\lambda_{\mathrm{r} 1 w}$, (f) variations of $k_{i}$ with respect to $\lambda_{\mathrm{r} 2 x}$, (g) variations of $k_{i}$ with respect to $\lambda_{\mathrm{r} 2 y}$, (h) variations of $k_{i}$ with respect to $\lambda_{\mathrm{r} 2 z}$, (i) variations of $k_{i}$ with respect to $\lambda_{\mathrm{r} 2 u}$, (j) variations of $k_{i}$ with respect to $\lambda_{\mathrm{r} 2 v}$, and $(\mathrm{k})$ variations of $k_{i}$ with respect to $\lambda_{\mathrm{r} 2 w}$.

\section{References}


[1] Li YM, Xu QS. Kinematic analysis of a 3-PRS parallel manipulator. Robotics and Computer-Integrated Manufacturing 2007; 23(4): 395-408.

[2] Hennes N, Staimer D. Application of PKM in aerospace manufacturing-high performance machining centers ECOSPEED, ECOSPEED-F and ECOLINER. In: Proceedings of the 4th Chemnitz Parallel Kinematics Seminar; 2004. p. 557-68.

[3] Hertel A. Requirements for parallel kinematics for powertrain manufacturing in automotive industry. In: Proceedings-3rd Chemnitz Parallel Kinematics Seminar. Zwickau, Germany; 2002. p. 753-762.

[4] Neumanm KE. Tricept application. In: Proceedings-3rd Chemnitz Parallel Kinematics Seminar. Zwickau, Germany; 2002. p. 547-551.

[5] Caccavale F, Siciliano B, Villani L. The Tricept robot: dynamics and impedance control. Mechatronics, IEEE/ASME Transactions on 2003; 8(2): 263-268.

[6] Weck M, Staimer D. Parallel kinematic machine tools-current state and future potentials. CIRP Annals Manufacturing Technology 2002; 51(2): 671-683.

[7] Fernandez AJS, Jimenez VC, Olazabal MG. Kinematical System for a Movable Platform of a Machine. European Patent(2002), No. EP1245349[A1].

[8] Ray P. Design of new high speed machining machines. Netherlands: Springer; 2005.

[9] Chen JS, Hsu WY. Design and analysis of a tripod machine tool with an integrated Cartesian guiding and metrology mechanism. Precision engineering 2004; 28(1): 46-57.

[10] Terrier M, Giménez M, Hascoet JY. VERNE-a five-axis parallel kinematics milling machine. Proceedings of the Institution of Mechanical Engineers, Part B: Journal of Engineering Manufacture 2005; 219(3): 327-336.

[11] Bi ZM, Jin Y. Kinematic modeling of Exechon parallel kinematic machine. Robotics and Computer-Integrated Manufacturing 2011; 27(1): 186-193.

[12] http://www.exechonworld.com/license.htm.

[13] Piras G, Cleghorn WL, Mills JK. Dynamic finite-element analysis of a planar high-speed, high-precision parallel manipulator with flexible links. Mechanism and Machine Theory 2005; 40(7): 849-862.

[14] Rizk R, Fauroux JC, Mumteanu M, Gogu G. A comparative stiffness analysis of a reconfigurable parallel machine with three or four degrees of mobility. Journal of machine engineering 2006; 6(2): 45-55.
[15] Wang YY, Liu HT, Huang T, Chetwynd DG. Stiffness modeling of the Tricept robot using the overall Jacobian matrix. Journal of Mechanisms and Robotics 2009; 1(2): 021002.

[16] Huang T, Zhao XY, Whitehouse DJ. Stiffness estimation of a tripod-based parallel kinematic machine. Robotics and Automation, IEEE Transactions on 2002; 18(1): $50-58$

[17] Ceccarelli M, Carbone G. A stiffness analysis for CaPaMan (Cassino parallel manipulator). Mechanism and Machine Theory 2002; 37(5): 427-439.

[18] Majou F, Gosselin C, Wenger P, Chablat D. Parametric stiffness analysis of the Orthoglide. Mechanism and Machine Theory 2007; 42(3): 296-311.

[19] Li YM, Xu QS. Stiffness analysis for a 3-PUU parallel kinematic machine. Mechanism and Machine Theory 2008; 43(2): 186-200

[20] Huang T, Liu HT, Chetwynd DG. Generalized Jacobian analysis of lower mobility manipulators. Mechanism and Machine Theory 2011; 46(6): 831-844.

[21] Huang S, Schimmels JM. The duality in spatial stiffness and compliance as realized in parallel and serial elastic mechanisms. Journal of dynamic systems, measurement, and control 2002; 124(1): 76-84.

[22] Dai JS, Ding XL. Compliance analysis of a three-legged rigidly-connected platform device. Journal of mechanical design 2006; 128(4): 755-764.

[23] Bonnemains T, Pateloup S, Chanal H, Bouzgarrou B, Ray P. Static behaviour analysis of a new parallel machine tool architecture: the Tripteor. In: Proceedings of IDMME - Virtual Concept. Beijing, China;, 2008. p. IDMME_50: $1-10$.

[24] Bonnemains T, Chanal H, Bouzgarrou BC, Ray P. Dynamic model of an overconstrained PKM with compliances: the Tripteor X7. Robotics and Computer-Integrated Manufacturing 2013; 29: 180-191.

[25] Li X, Zlatanov D, Zoppi M, Molfino R. Stiffness estimation and experiments for the Exechon parallel self-reconfiguring fixture mechanism. In: ASME 2012 International Design Engineering Technical Conferences and Computers and Information in Engineering Conference. American Society of Mechanical Engineers; 2012. p. 637-645.

[26] Bi ZM. Kinetostatic modeling of Exechon parallel kinematic machine for stiffness analysis. The International Journal of Advanced Manufacturing Technology 2014; 71(1-4): 325-335. 
[27] Li YG, Liu HT, Zhao XM, Huang T, Chetwynd DG. Design of a 3-DOF PKM module for large structural component machining. Mechanism and Machine Theory 2010; 45(6): 941-954. 